AperTO - Archivio Istituzionale Open Access dell'Università di Torino

A cellular Potts model analyzing differentiated cell behavior during in vivo vascularization of a hypoxic tissue

This is a pre print version of the following article:

Original Citation:

Availability:

This version is available http://hdl.handle.net/2318/1520762

since 2015-12-23T11:27:26Z

Published version:

DOI:10.1016/j.compbiomed.2015.05.020

Terms of use:

Open Access

Anyone can freely access the full text of works made available as "Open Access". Works made available under a Creative Commons license can be used according to the terms and conditions of said license. Use of all other works requires consent of the right holder (author or publisher) if not exempted from copyright protection by the applicable law. 


\section{(6) \\ UNIVERSITÀ DEGLI STUDI DI TORINO}

This is an author version of the contribution published on:

Questa è la versione dell'autore dell'opera:

Comput Biol Med. 2015 Aug;63:143-56. doi: 10.1016/j.compbiomed.2015.05.020. Epub 2015 Jun 3.

The definitive version is available at:

La versione definitiva è disponibile alla URL:

http://www.sciencedirect.com/science/article/pii/S0010482515002024 


\title{
A cellular Potts model analyzing differentiated cell behavior during in vivo vascularization of a hypoxic tissue
}

\author{
Marco Scianna ${ }^{1}$ \\ Department of Mathematics, Politecnico di Torino, Corso Duca degli Abruzzi 24, 10129 \\ Torino, Italy \\ Eleonora Bassino ${ }^{2}$ and Luca Munaron ${ }^{3}$ \\ Department of Life Sciences and Systems Biology, University of Torino, Via Accademia \\ Albertina 13, 10123, Torino, Italy
}

\begin{abstract}
Angiogenesis, the formation of new blood vessel networks from existing capillary or post-capillary venules, is an intrinsically multiscale process occurring in several physio-pathological conditions. In particular, hypoxic tissue cells activate downstream cascades culminating in the secretion of a wide range of angiogenic factors, including VEGF isoforms. Such diffusive chemicals activate the endothelial cells (ECs) forming the external walls of the nearby vessels, that chemotactictally migrate toward the hypoxic areas of the tissue as multicellular sprouts. A functional network eventually emerges by further branching and anastomosis processes. We here propose a CPM-based approach reproducing selected features of the angiogenic progression necessary for the reoxygenation of a hypoxic tissue. Our model is able to span the different scale involved in the angiogenic progression as it incorporates reaction-diffusion equations for the description of the evolution of microenvironmental variables in a discrete mesoscopic cellular Potts model (CPM) that reproduces the dynamics of the vascular cells. A key feature of this work is the explicit phenotypic differentiation of the ECs themselves, distin-
\end{abstract}

\footnotetext{
${ }^{1}$ E-Mail: marcosci1@alice.it

${ }^{2}$ E-Mail: eleonora.bassino@unito.it

${ }^{3}$ E-Mail: luca.munaron@unito.it
} 
guished in quiescent, stalk and tip. The simulation results allow identifying a set of key mechanisms underlying tissue vascularization. Further, we provide evidence that the nascent pattern is characterized by precise topological properties. Finally, we link abnormal sprouting angiogenesis with alteration in selected cell behavior.

Keywords: multiscale model, angiogenesis, capillary network, delta-notch signaling, oxygenation of hypoxic tissues

\section{Introduction}

Blood vessel formation and development involves two different mechanisms: vasculogenesis and angiogenesis (for comprehensive reviews, see $[6,9,10])$. Vasculogenesis consists of the de novo formation of a primitive vascular network, that emerges from migration, aggregation and organization of typically-dispersed populations of endothelial cells (ECs). Angiogenesis refers instead to the formation of new vessels from an existing capillary or post-capillary venule. Although angiogenesis intervenes at the embryonic stage to remodel the initial capillary network into a mature and functional vascular bed (comprised of arteries, capillaries, and veins), its main role is played during adult life, when it is involved in many physiological processes, as the vascularization of hypoxic tissues, of ovary and uterus during the female cycle, of mammary gland during lactation, and of granulation tissue during wound healing. However, when the equilibrium of its underlying control mechanisms is disrupted, angiogenesis becomes pathological, as in the case of chronic inflammatory diseases, vasculopathies, degenerative disorders, and tissue injury occurring in ischemia. Finally, angiogenic progression is a pivotal transition phase in the development of cancer: in fact, by providing nutrition and oxygen, it allows malignant cells to grow and remain viable, and, eventually, to spread metastases through invasion of the circulatory system $[9,10]$.

Entering in more details, angiogenesis is a multiscale program which involves mechanisms at both the subcellular and the cellular level. The overall process starts when tissue cells, deprived of oxygen and/or nutrients, accumulate in the nucleus increasing amounts of hypoxic growth factor (HIF-1). By selected binding to DNA, HIF-1 is able to regulate the expression levels of numerous genes, which control the production of a wide range of angiogenic growth factors $[13,29]$, including vascular endothelial growth factors 
(VEGFs), transforming growth factor $\beta$ (TGF- $\beta$ ), basic fibroblast growth factor (bFGF), platelet-derived growth factor (PDGF). Such diffusive chemicals in turn stimulate the endothelial cells forming the external walls of preexisting blood vessels, which loosen their adhesive connections, thereby reducing the vascular tonus and increasing both the permeability the interstitial pressure. The activated ECs are then able to migrate chemotactically towards the hypoxic areas of tissues (i.e., those releasing angiogenic factors) as multicellular strands. Collective EC movement is facilitated by the production of proteolytic enzymes (serine-proteins, iron-proteins, matrix metalloproteinases) which degrade the basal lamina and the extracellular matrix surrounding the growing branches. An optimal extension of new vascular sprouts is allowed by a phenotypic differentiation of the component ECs, which undergo the so-called tip cell selection and lateral inhibition, which is mediated by VEGF-induced delta-notch signaling pathways. This process occurs in the following stages: i) activation of VEGFR-2 receptors in a tip individual leads to up-regulation of ligand Dll4 [36]; ii) Dll4, in turn, is able to activate Notch1 receptors in the neighboring cells, whose VEGFR-2 expression is consequently down-regulated. The Notch1-overexpressing individuals are set to assume a stalk fate [51, 72]. The notch-Dll4 lateral inhibition generates therefore an alternating pattern of cell phenotype, a salt and pepper pattern [8], where tip cells are separated by one or two stalk cells. During angiogenic progression, tip individuals lead the way through the extracellular space (also by producing matrix degrading enzymes) while stalk cells, forming the rear of the multicellular strands, sustain branch elongation by repeated divisions along the axis perpendicular to the direction of sprout extension. During growth, vascular sprouts undergo branching or they encounter and merge to form loops, a process called anastomosis. From these branches and loops more sprouts form. Eventually, the whole process repeats several times, resulting in the formation of a capillary-like network. In a subsequent maturation stage, the new born vessels are remodelled into a more elaborate and hierarchically-spaced vascular tree by pruning events resulting in the loss of some physiologically useless capillaries. The last phase of the angiogenic programme involves the formation and size-adaptation of the capillary lumen, the formation of new perivascular ECM and the arrival of pericytes and sometimes of flat muscle cells.

Experimental studies performed on in vivo systems have revealed the role of many different factors driving the formation of vascular networks via sprouting angiogenesis in physiological situations. However innumer- 
able other processes, acting at different spatiotemporal scales, remain far from being completely understood. The complexity of the problem makes in fact it difficult and expensive to study using solely laboratory-based biological methods: indeed, the support and insight gained by using in silico approaches is vital. For the sake of completeness, we have to admit that, although angiogenesis occurs in a wide range of physio-pathological circumstances and situations, the majority of the theoretical literature focuses on the tumor-induced angiogenesis, one of the most dangerous aspects, as reviewed in [58]. However, in this work, we propose a multilevel mathematical model reproducing an in vivo revascularization and reoxygenation of a hypoxic tissue. The endothelial cell population is described at the mesoscopic level with a discrete cellular Potts model (CPM), a lattice-based Monte Carlo technique which follows an energy minimization philosophy and preserves the identity and the behaviors of single individuals [21, 22, 23, 24, 55, 61]. In particular, cell exploratory movements are implemented by stochastic acceptances of domain configuration updates, which depend on sets of cell physical and behavioral rules. Selected reaction-diffusion (RD) equations instead describe the evolution of molecular variables, i.e., angiogenic growth factors and oxygen. These two components are integrated and interfaced together, constituting a hybrid and multiscale simulation environment characterized by a constant flux of information from the different levels, as the kinetics of the microscopic variables strongly influence cell dynamics. Our model appears a possibly new and biologically interesting representative in the class of models of blood vessel development: we here in fact focus on the role played by the dynamical and self-emerging differentiation of phenotypes of both vascular cells (i.e., quiescent, tip, stalk) and of tissue cells (oxygenated, hypoxic). As we will see in the following, the model results will reproduce with good accuracy the emerging of a functional capillary network, able to provide the reoxygenation of the host tissue. Then, through different sets of numerical realizations, we will dissect selected component mechanisms of the angiogenic progression, in order to link altered newly-formed vascular structures with abnormal cell behavior.

The rest of this paper is organized as follows. In Section 2, we clarify the assumptions on which our approach is based and present the model components. The simulation results are then shown in Section 3. Finally, a discussion on possible model improvements as well as a comparison with the existing theoretical literature on these topics is proposed in Section 4. 


\section{Mathematical model}

Model assumptions. The major hypothesis of the model, which account both intracellular biochemical cascades and cell-level behavior, include: 1) we start with a tissue characterized by a preexisting, but insufficient, vasculature. In particular, we neglect the distinction between veins and arteries; 2) oxygen-depleted areas of a tissue become hypoxic by simple thresholding depending on the local partial pressure of oxygen; 3) hypoxic tissue starts to secrete a single long-diffusion-length isoform of VEGF-A, like VEGF-A ${ }_{120,121}$. The molecular HIF-1-dependent cascades resulting in VEGF-A production are not explicitly included in the model; 4) VEGF-A activity induces a dosedependent activation of the ECs forming the external walls of the preexisting vessels; 5) we include tip cell selection and stalk cell lateral inhibition without explicitly modeling the delta-notch signaling pathways; 6) the tip cells respond to VEGF-A by polarizing and chemotactically migrating, while the stalk individuals respond by proliferating [13]; 7) the tip/stalk cell fate is reversible, in the sense that a tip cell can assume a stalk state after anastomosis and a stalk cell can acquire a tip fate if it is not surrounded by tip individuals. In this last case, we do not consider recovery delays, which are due to the time needed by stalk cell gene expression to return in a normal non-inhibited state; 8) both type of activated vascular endothelial cells secrete and chemotax towards a short-diffusion-length chemoattractant (which can represent for instance VEGF-A $165[60])$; 9) oxygen is assumed to be secreted both by the functional preexisting vasculature and by emerging loop structures.

Extended cellular Potts model. The physiological angiogenic process is modeled at the mesoscopic level using an extended version of the cellular Potts model, a grid-based stochastic approach, which realistically preserves the identity of single cell-level individuals and describes their behavior and interactions with the environment in energetic terms and constraints. The simulation domain is a two-dimensional regular lattice $\Omega \subseteq \mathbb{R}^{2}$, formed by identical closed grid sites that, with an abuse of notation, will be identified by their center $\mathbf{x} \in \mathbb{R}^{2}$. Each site is labeled by an integer number, $\sigma(\mathbf{x}) \in \mathbb{N}$, that can be interpreted as a degenerate spin originally coming from statistical physics $[27,48]$. As classically adopted in CPMs, a neighboring site of $\mathbf{x}$ is denoted by $\mathbf{x}^{\prime}$, while its overall neighborhood by $\Omega_{\mathbf{x}}^{\prime}$, i.e., $\Omega_{\mathbf{x}}^{\prime}=\left\{\mathbf{x}^{\prime} \in \Omega: \mathbf{x}^{\prime}\right.$ is a neighbor of $\mathbf{x}$. A vascular cell, identified by $\Sigma_{\sigma}$, consists of a subdomain 
of contiguous sites with identical spin (i.e., $\Sigma_{\bar{\sigma}}=\{\mathbf{x} \in \Omega: \sigma(\mathbf{x})=\bar{\sigma}\}$ with $\bar{\sigma}=1, \ldots, N(t)$, where $N(t)$ is the total number of ECs at time $t$ ), and has an associated type $\tau\left(\Sigma_{\sigma}\right)$. In particular, we here distinguish three types of vascular cells: quiescent, $\tau=Q$, tip, $\tau=T$, and stalk, $\tau=S$. Both the tip and the stalk phenotype can be classified as activated. We further define a special, generalized cell $\Sigma_{\sigma=0}$ representing the host tissue, which is indeed formed by the part of the domain not occupied by any endothelial cell. Such an extravasal space is assumed to be isotropically distributed, forming no large-scale structures: however, it can locally assume two different states: oxygenated, $\tau=O$ (i.e., normoxic), and hypoxic, $\tau=H$. It is useful to remark that, in this case, the type attribute $\tau$ is defined for every site (i.e., $\tau=\tau(\mathbf{x}))$ whereas, in the case of vascular cells, it identifies the entire cluster of sites forming a single individual (i.e., $\tau=\tau\left(\Sigma_{\sigma}\right)$ ).

Cell movement results from an iterative and stochastic reduction of the energy of the overall system, given by a hamiltonian $H$, whose expression will be clarified below. The core algorithm consists of elementary steps of a modified Metropolis method for Monte Carlo-Boltzmann dynamics [24, 39]. This approach is particularly suitable to implement the natural exploratory behavior of biological individuals. Procedurally, at each step $t$ of the algorithm, called Monte Carlo Step (MCS), a lattice site $\mathbf{x}_{\mathbf{s}}$ (s for source) is selected at random and attempts to copy its spin, $\sigma\left(\mathbf{x}_{\mathrm{s}}\right)$, into one of its unlike neighbors, $\mathbf{x}_{\mathrm{t}} \in \Omega_{\mathbf{x}_{\mathrm{s}}}^{\prime}: \mathbf{x}_{\mathrm{t}} \notin \Sigma_{\sigma\left(\mathbf{x}_{\mathrm{s}}\right)}$ (t for target), also randomly selected. In particular, if $\tau\left(\Sigma_{\sigma\left(\mathbf{x}_{\mathrm{s}}\right)}\right)=\{Q, S, T\}$, the cell $\Sigma_{\sigma\left(\mathbf{x}_{\mathrm{s}}\right)}$ is protruding (i.e., extending its filopods towards another cell or in the extracellular space). Otherwise, if $\sigma\left(\mathbf{x}_{\mathrm{s}}\right)=0$ (i.e., $\left.\tau\left(\mathbf{x}_{\mathrm{s}}\right)=\{O, H\}\right)$, the cell $\Sigma_{\sigma\left(\mathbf{x}_{\mathrm{t}}\right)}$ is retracting.

Each trial spin update is accepted with a Boltzmann-like probability function $P\left(\sigma\left(\mathbf{x}_{\mathrm{s}}\right) \rightarrow \sigma\left(\mathbf{x}_{\mathrm{t}}\right)\right)$, that involves both a symmetric minimization of the system free energy and an asymmetric bias relating to the specific behavior 
of tip individuals:

$$
P\left(\sigma\left(\mathbf{x}_{\mathbf{s}}\right) \rightarrow \sigma\left(\mathbf{x}_{\mathrm{t}}\right)\right)(t)=\left\{\begin{array}{c}
\tanh \left(T_{\Sigma_{\sigma\left(\mathbf{x}_{\mathbf{s}}\right)}}(t)\right) \min \left\{1, \exp \left(\frac{-\Delta H+w_{\text {pol }}}{T_{\Sigma_{\sigma\left(\mathbf{x}_{\mathbf{s}}\right)}}(t)}\right)\right\} \\
\text { if } \tau\left(\Sigma_{\sigma\left(\mathbf{x}_{\mathrm{s}}\right)}\right)=T ; \\
\tanh \left(T_{\Sigma_{\sigma\left(\mathbf{x}_{\mathbf{s}}\right)}}(t)\right) \min \left\{1, \exp \left(\frac{-\Delta H}{T_{\Sigma_{\sigma\left(\mathbf{x}_{\mathbf{s}}\right)}}(t)}\right)\right\} \\
\text { if } \tau\left(\Sigma_{\sigma\left(\mathbf{x}_{\mathbf{s}}\right)}\right)=\{Q, S\} ; \\
\tanh \left(T_{\Sigma_{\sigma\left(\mathbf{x}_{\mathrm{t}}\right)}}(t)\right) \min \left\{1, \exp \left(\frac{-\Delta H}{T_{\Sigma_{\sigma\left(\mathbf{x}_{\mathrm{t}}\right)}}(t)}\right)\right\} \\
\text { if } \sigma\left(\mathbf{x}_{\mathrm{s}}\right)=0,
\end{array}\right.
$$

where $\Delta H$ is the net difference of the hamiltonian due to the proposed change of domain configuration, $w_{\text {pol }}$ describes the directional persistent movement of polarized cells (see below), and $T_{\Sigma_{\sigma(\mathbf{x})}}(t) \in R_{+}$, where $\mathbf{x} \in\left\{\mathbf{x}_{\mathrm{s}}, \mathbf{x}_{\mathrm{t}}\right\}$, is a Boltzmann temperature. As commented in [55], the specific form of the transition probability (1) is identified so that it accounts also cells with a substantially null motility, i.e., individuals for which the probability of moving is negligible even in the presence of favorable energy gradients. In this respect, $T_{\Sigma_{\sigma(\mathbf{x})}}$ measures the agitation rate of moving (i.e., either retracting or extending) cell $\Sigma_{\sigma(\mathbf{x})}$. In other words, it is a measure of the intrinsic cell motility, i.e., of the frequency of membrane ruffles which, on a molecular level, are determined by polarization/depolarization processes of the actin cytoskeleton, enhanced in a dose-dependent manner by the intracellular level 
of growth factors:

$$
T_{\Sigma_{\sigma}}(t)=\left\{\begin{array}{llr}
T_{Q} & \text { if } & \tau\left(\Sigma_{\sigma}\right)=Q ; \\
T_{Q}\left[\frac{1+v\left(\Sigma_{\sigma}, t\right)}{1+h v\left(\Sigma_{\sigma}, t\right)} \frac{1+c\left(\Sigma_{\sigma}, t\right)}{1+h c\left(\Sigma_{\sigma}, t\right)}\right] & \text { if } & \tau\left(\Sigma_{\sigma}\right)=T ; \\
T_{Q}\left[\frac{1+c\left(\Sigma_{\sigma}, t\right)}{1+h c\left(\Sigma_{\sigma}, t\right)}\right] & \text { if } & \tau\left(\Sigma_{\sigma}\right)=S,
\end{array}\right.
$$

where $T_{Q}$ corresponds to the basal motility typical of resting ECs. In particular, $v\left(\Sigma_{\sigma}, t\right)=\sum_{\mathbf{x} \in \Sigma_{\sigma}} v(\mathbf{x}, t)$ and $c\left(\Sigma_{\sigma}, t\right)=\sum_{\mathbf{x} \in \Sigma_{\sigma}} c(\mathbf{x}, t)$ measure the total amounts inside cell $\Sigma_{\sigma}$ of the tissue-secreted VEGF-A and of the ECproduced chemoattractant, respectively, as $v(\mathbf{x}, t)$ and $c(\mathbf{x}, t)$ are their local concentrations defined in Eqs. (12) and (13). In particular, in (2), we account the fact that the short-range autocrine chemical induces a migratory response in both tip and stalk cells, whereas the long-range VEGF-A only in tip ECs (due to the delta/notch-mediated lateral inhibition of VEGFdependent motility in stalk individuals).

The term $w_{\text {pol }}$, previously introduced in CPMs describing formation and growth of elongated multicellular structures [66, 67, 68], accounts the polarization of tip vascular cells (i.e., their ability to differentiate in a leading and a trailing edge) and the resulting persistent directional migration, which alters the probability assigned to each spin update, as:

$$
w_{\mathrm{pol}}\left(\sigma\left(\mathbf{x}_{\mathrm{s}}\right) \rightarrow \sigma\left(\mathbf{x}_{\mathrm{t}}\right)\right)=P \frac{\mathbf{p}_{\Sigma_{\sigma\left(\mathbf{x}_{\mathrm{s}}\right)}}}{\left|\mathbf{p}_{\Sigma_{\sigma\left(\mathbf{x}_{\mathrm{s}}\right)}}\right|} \cdot\left(\mathbf{x}_{\mathrm{t}}-\mathbf{x}_{\mathrm{s}}\right),
$$

where $P$ sets the magnitude of the cell persistent movement and $\mathbf{p}_{\Sigma_{\sigma\left(\mathbf{x}_{\mathbf{s}}\right)}}$ is the polarity vector of the extending tip cell $\Sigma_{\sigma\left(\mathbf{x}_{\mathrm{s}}\right)} \cdot \mathbf{p}_{\Sigma_{\sigma\left(\mathbf{x}_{\mathrm{s}}\right)}}$ is updated by considering a spontaneous decay and reinforcement from cell displacements. In fact, for each MCS and for any tip cell $\Sigma_{\sigma}$ (i.e., $\tau\left(\Sigma_{\sigma}\right)=T$ ), we have that

$$
\Delta \mathbf{p}_{\Sigma_{\sigma}}=-\frac{1}{t_{\mathbf{p}}} \mathbf{p}_{\Sigma_{\sigma}}+\Delta \mathbf{x}_{\Sigma_{\sigma}}^{C M}
$$

where $t_{\mathbf{p}}$ is a characteristic memory length of the polarization vector, which can be also defined as the inverse of a rate of spontaneous decay, and $\Delta \mathbf{x}_{\Sigma_{\sigma}}^{C M}$ is the net displacement of the cell center of mass during the MCS considered. 
For any given step $t$ of the algorithm, the energy of the biological environment, whose minimization, as seen, drives the evolution of the system, is determined by the sum of different contributions:

$$
H(t)=H_{\text {shape }}(t)+H_{\text {adhesion }}(t)+H_{\text {chemotaxis }}(t) .
$$

$H_{\text {shape }}$ models the geometrical attributes of vascular cells, which are written as non-dimensional relative deformations in the following quadratic form (see [55] for a more detailed explanation):

$$
\begin{aligned}
H_{\text {shape }}(t) & =H_{\text {surface }}(t)+H_{\text {perimeter }}(t) \\
& =\sum_{\Sigma_{\sigma}}\left[\kappa_{\Sigma_{\sigma}}(t)\left(\frac{s_{\Sigma_{\sigma}}(t)-S_{E C}}{s_{\Sigma_{\sigma}}(t)}\right)^{2}+\nu_{\Sigma_{\sigma}}(t)\left(\frac{p_{\Sigma_{\sigma}}(t)-P_{E C}}{p_{\Sigma_{\sigma}}(t)}\right)^{2}\right](6)
\end{aligned}
$$

which depend on their actual surface and perimeter (we recall that here the model is two-dimensional, but the 3D-extension is straightforward), $s_{\Sigma_{\sigma}}(t)$ and $p_{\Sigma_{\sigma}}(t)$, as well as on the same quantities in the relaxed state, $S_{E C}$ and $P_{E C}$, which correspond instead to their initial measures. $\kappa_{\Sigma_{\sigma}}(t)$ and $\nu_{\Sigma_{\sigma}}(t) \in$ $\mathbb{R}_{+}$are mechanical moduli in units of energy: in particular, $\kappa_{\Sigma_{\sigma}}(t)$ refers to cell surface changes, while $\nu_{\Sigma_{\sigma}}(t)$ relates to cell deformability/elasticity, i.e., the ease with which an individual is able to remodel changing its perimeter. Assuming that all ECs have an adequate amount of intracellular nutrients and that they do not significantly grow during migration, the surface fluctuations are kept negligible with a high constant value $\kappa_{\Sigma_{\sigma}}(t)=\kappa \gg 1$, for any cell $\Sigma_{\sigma}$ regardless of its type. We instead establish specific elastic properties for each vascular cell phenotype. In particular, quiescent ECs have a constantly high rigidity, which is fundamental to preserve the structure and the functionality of mature vessels. On the opposite, cytoskeletal reorganizations of stalk and tip individuals are stimulated by autocrine and paracrine angiogenic factors. Following these considerations, we set

$$
\nu_{\Sigma_{\sigma}}(t)=\left\{\begin{array}{lll}
\nu_{Q} & \text { if } & \tau\left(\Sigma_{\sigma}\right)=Q \\
\nu_{Q} \exp \left(-k\left[v\left(\Sigma_{\sigma}, t\right)+c\left(\Sigma_{\sigma}, t\right)\right]\right) & \text { if } & \tau\left(\Sigma_{\sigma}\right)=T \\
\nu_{Q} \exp \left(-k c\left(\Sigma_{\sigma}, t\right)\right) & \text { if } & \tau\left(\Sigma_{\sigma}\right)=S
\end{array}\right.
$$

where $\nu_{Q}$ is the intrinsic cells' resistance to compression and $v\left(\Sigma_{\sigma}, t\right), c\left(\Sigma_{\sigma}, t\right)$ are defined as in Eq. (2). Also in this case, we account the notch-mediated 
stalk cell inhibition of VEGF-A migratory response. In particular, we use negative exponential functions because very high levels of motility factors promote continuous and dramatic actin-myosin interactions, resulting in quick changes in cell organization and shape, as widely provided in the experimental literature. Finally, it is useful to remark that the high rigidity of quiescent EC (i.e., $\nu_{Q} \gg 1$ ) is fundamental to maintain a stable structure of the preexisting vasculature.

$H_{\text {adhesion }}$, deriving from the Steinberg's Differential Adhesion Hypothesis (DAH) $[24,64,65]$, measures the adhesive interactions between different cells or between a cell and the host tissue:

$$
H_{\text {adhesion }}(t)=\sum_{\mathbf{x} \in \Sigma_{\sigma}, \mathbf{x}^{\prime} \in \Sigma_{\sigma^{\prime}}} J_{\tau\left(\Sigma_{\sigma(\mathbf{x})}\right), \tau\left(\Sigma_{\sigma^{\prime}\left(\mathbf{x}^{\prime}\right)}\right)}(t),
$$

where $\mathbf{x}$ and $\mathbf{x}^{\prime}$ are two neighboring sites and $\Sigma_{\sigma}$ and $\Sigma_{\sigma^{\prime}}$, where $\sigma \neq \sigma^{\prime}$, two neighboring elements. The coefficients $J_{\tau\left(\Sigma_{\sigma}\right), \tau\left(\Sigma_{\sigma^{\prime}}\right)} \in \mathbb{R}_{+}$are binding forces per unit area and are obviously symmetric w.r.t. the indices. In particular, by setting constant and homogeneous values for such parameters, we here assume a uniform distribution of adhesion molecules on the cell surface and a homogenous density of ligands in the external microenvironment. In order to establish a consistent hierarchy of the cell adhesive forces, we first observe that vascular tonus and functionality are determined by strong intercellular connections. On the opposite, angiogenic processes are permitted, in the first stages, by the loosening of cell-cell adhesiveness occurring between VEGFactivated ECs (both stalk and tip), which allows them to reorganize and eventually migrate. We finally suppose that vascular cells (regardless of their phenotype) prefer to adhere to one another rather than to the host. The above considerations lead to the following relations:

$$
\begin{aligned}
J_{Q, Q}<J_{Q, T} & =J_{Q, S}<J_{S, S}=J_{S, T}=J_{T, T}<J_{Q, O} \\
& =J_{Q, H}=J_{S, O}=J_{S, H}=J_{T, O}=J_{T, H} .
\end{aligned}
$$

$H_{\text {chemotaxis }}$ reproduces the effect of cell preferential migration in the direction of zones with higher concentration of diffusing chemoattractants. In particular, we here account the tip cell movement along gradients of longrange diffusing VEGF-A and the migratory response of both tip and stalk individuals to the autocrine chemical field. Such energy contributions are implemented by local linear-type relations of the form used in [52] for the 
chemotactic processes in Dictyostelium Discoideum aggregation:

$$
\begin{aligned}
\Delta H_{\text {chemotaxis }} & =\mu_{v, \Sigma_{\sigma\left(\mathbf{x}_{\mathbf{s}}\right)}}\left[v_{t}\left(\mathbf{x}_{\mathrm{t}}, t\right)-v_{t}\left(\mathbf{x}_{\mathbf{s}}, t\right)\right] \\
& +\mu_{c, \Sigma_{\sigma\left(\mathbf{x}_{\mathbf{s}}\right)}}(t)\left[c_{t}\left(\mathbf{x}_{\mathrm{t}}, t\right)-c_{t}\left(\mathbf{x}_{\mathbf{s}}, t\right)\right],
\end{aligned}
$$

where $\mathbf{x}_{\mathrm{s}}$ and $\mathbf{x}_{\mathrm{t}}$ are, respectively, the source and the final lattice site randomly selected during a trial update in a MCS, and $v_{t}(\mathbf{x}, t)=v(\mathbf{x}, t)+$ $\sum_{\mathbf{x}^{\prime} \in \Omega_{\mathbf{x}}^{\prime}} v\left(\mathbf{x}^{\prime}, t\right)$ and $c_{t}(\mathbf{x}, t)=c(\mathbf{x}, t)+\sum_{\mathbf{x}^{\prime} \in \Omega_{\mathbf{x}}^{\prime}} c\left(\mathbf{x}^{\prime}, t\right)$, where $\mathbf{x} \in\left\{\mathbf{x}_{\mathbf{s}}, \mathbf{x}_{\mathrm{t}}\right\}$, evaluate the local level of chemical factors sensed by the moving cell membrane site. Finally, $\mu \in \mathbb{R}^{+}$represent the strength of chemotactic response: in particular, $\mu_{v, \Sigma_{\sigma\left(\mathbf{x}_{\mathbf{s}}\right)}}=\mu_{v}$ for $\tau\left(\Sigma_{\sigma\left(\mathbf{x}_{\mathrm{s}}\right)}\right)=T$ (and $=0$ else), whereas $\mu_{c, \Sigma_{\sigma\left(\mathbf{x}_{\mathrm{s}}\right)}}=\mu_{c}<\mu_{v}$ for $\tau\left(\Sigma_{\sigma\left(\mathbf{x}_{\mathrm{s}}\right)}\right)=\{T, S\}$ (and $=0$ else). We finally implement a contact-inhibition of chemotaxis by setting $\Delta H_{\text {chemotaxis }}=0$ at the cell-cell interfaces (see [37]).

Stalk cell proliferation. As suggested by a number of experimental works as early as [63], the time between cell divisions has a stochastic distribution, which depends on both the internal state of each cell and on the time from its last mitotic process (i.e., except for extremely rapidly dividing cells, whose likelihood of reentering the $\mathrm{S}$ phase is extremely small, see [1]). In our case, the proliferation of stalk cells, fundamental for sprout elongation, is regulated by long-range diffusing VEGF-A activity (which, instead, do not influence their migratory response due to the delta/notch-mediated lateral inhibition, as previously seen). For each cell $\Sigma_{\sigma}$ such as $\tau\left(\Sigma_{\sigma}\right)=S$, we therefore define the present probability $P_{\Sigma_{\sigma}}$ for cell $\Sigma_{\sigma}$ to undergo mitosis with the following functional form, which resembles that used in similar approaches [54,70]:

$$
P_{\Sigma_{\sigma}}(t)=\left\{\begin{array}{lll}
0 & \text { if } & \left(t-t_{\Sigma_{\sigma}}\right) \leq t_{0} ; \\
\frac{v\left(\Sigma_{\sigma}, t\right)}{1+v\left(\Sigma_{\sigma}, t\right)} \frac{\left(t-t_{\Sigma_{\sigma}}\right)^{2}}{1+\left(t-t_{\Sigma_{\sigma}}\right)^{2}} & \text { if } & \left(t-t_{\Sigma_{\sigma}}\right)>t_{0} .
\end{array}\right.
$$

$t_{\Sigma_{\sigma}}$ is the last time that cell $\Sigma_{\sigma}$ underwent mitosis, while $t_{0}$ is a dormant period during which the cells are prohibited to proliferate (i.e., it correspond to the G1 phase, during which the cells are metabolically active and grow). Procedurally, the mechanism of cell division is implemented by dividing the proliferating individual into two identical daughter cells with a halved area with respect to their parent: however, due to the shape constraints in Eq. (6), they will gradually "maturate" into full-size cells. We further assume 
that both daughter cells evenly inherit all the parent's biophysical properties, i.e., stalk phenotype, motility and elasticity. Finally, the newly formed individuals are placed symmetrically about the parent cell center of mass with the same orientation of the elongating sprout. This is of particular relevance, since the orientation of stalk cell division has been widely provided to be a critical factor for angiogenic processes [20].

Transition between cell phenotypes. The host tissue can locally switches between the oxygenated (i.e., normoxic) and the hypoxic phenotype according to the local partial pressure of oxygen. In particular, a tissue site in the oxygenated state becomes hypoxic when the local oxygen partial pressure drops below a given threshold $o_{p r, h}$. It can then return to the oxygenated state when the oxygen partial pressure increases again beyond this level. Quiescent vascular cells acquire an activated phenotype (i.e., either tip or stalk) in the presence of a level of tissue-secreted VEGF-A greater than the given threshold $v_{a}$. In particular, when a quiescent EC becomes tip, its first-nearer neighboring individuals receive the lateral inhibition and become stalk. Stalk cells can in turn acquire a tip fate as soon as they do not have any adjacent tip individuals. When two tip cells join together (i.e., with an anastomosis process), the tip individual with the higher level of VEGF-A does not undergo a phenotypic transition, whereas the other is inhibited and receives a stalk state. Finally, both tip and stalk cells stall and return back to a quiescent phenotype when their amount of VEGF-A drops below the activating level $v_{a}$. The proposed rules for transition between cell phenotypes give a further multiscale characteristic of our approach, as the cell mesoscopic phenotype is determined by specific microscopic events.

Evolution of molecular variables. The microscopic level of the system is described by a set of reaction-diffusion (RD) equations. Long-range diffusing VEGF-A is secreted by hypoxic tissue at a constant rate, diffuses and decays homogeneously within the entire domain, and is uptaken by vascular cells. Its spatial profile therefore satisfies the following equation:

$$
\begin{aligned}
\frac{\partial v(\mathbf{x}, t)}{\partial t} & =\underbrace{D_{v} \Delta v(\mathbf{x}, t)}_{\text {diffusion }}-\underbrace{\lambda_{v} v(\mathbf{x}, t)}_{\text {decay }}-\underbrace{\min \left\{v_{m}, \chi_{v} v(\mathbf{x}, t)\right\} \delta_{\tau\left(\Sigma_{\sigma(\mathbf{x})}\right),\{Q, T, S\}}}_{\text {uptake }} \\
& +\underbrace{\alpha_{v} \delta_{\tau\left(\Sigma_{\sigma(\mathbf{x})}\right), H}}_{\text {production }},
\end{aligned}
$$


where $\delta_{x, y}=\{1, x=y ; 0, x \neq y\}$ is the Kronecher delta. The third term at the r.h.s. models the VEGF-A absorption by all type of vascular cells, which follows a piecewise-linear approximation of a Michaelis-Menten law (this simplification is realistic since cell capacity to absorb chemicals typically saturates to a limit established both by the density of membrane-bound receptors and by the rate at which the chemical substance can be internalized and the receptors recycled). In particular, $\lambda_{v} \ll \chi_{v}$, as we assume that the VEGF-A natural decay is negligible compared to the EC uptake.

The autocrine, short-diffusing angiogenic factor is secreted by both tip and stalk cells. It then degrades and diffuses at a constant rate everywhere and is finally absorbed by the activated vascular individuals themselves:

$$
\begin{aligned}
\frac{\partial c(\mathbf{x}, t)}{\partial t} & =\underbrace{D_{c} \Delta c(\mathbf{x}, t)}_{\text {diffusion }}-\underbrace{\lambda_{c} c(\mathbf{x}, t)}_{\text {decay }}-\underbrace{\min \left\{c_{m}, \chi_{c} c(\mathbf{x}, t)\right\} \delta_{\tau\left(\Sigma_{\sigma(\mathbf{x})}\right),\{T, S\}}}_{\text {uptake }} \\
& +\underbrace{\alpha_{c} \delta_{\tau\left(\Sigma_{\sigma(\mathbf{x})}\right),\{T, S\}}}_{\text {production }} .
\end{aligned}
$$

Also in this case we use an approximate version of a Michaelis-Menten law for chemical uptake, with $\lambda_{c} \ll \chi_{c}$.

The oxygen partial pressure at location $\mathbf{x}$ is defined by a field $o_{p r}(\mathbf{x}, t)$. This implies that the local available concentration of oxygen is proportional to $o_{p r}$, i.e., $O_{2}(\mathbf{x}, t)=\xi_{o} o_{p r}(\mathbf{x}, t)$, where $\xi_{o}$ is a solubility coefficient. Following [62], we assume that oxygen diffuses at a constant rate throughout the simulation domain and it is consumed by the tissue. Indeed, oxygen partial pressure locally evolves according to the following reaction-diffusion equation:

$$
\frac{\partial o_{p r}(\mathbf{x}, t)}{\partial t}=\underbrace{\xi_{o} D_{o} \Delta o_{p r}(\mathbf{x}, t)}_{\text {diffusion }}-\underbrace{\min \left\{o_{m}, \chi_{o} o_{p r}(\mathbf{x}, t)\right\} \delta_{\tau(\mathbf{x}),\{O, H\}}}_{\text {consumption }},
$$

where the oxygen consumption by the tissue is limited to a maximum rate (i.e., for the sake of simplicity we do not include oxygen absorption by the ECs themselves). Oxygen supply is assured at given partial pressures both from the preexisting vessels and from loop structures formed in the emerging 
network by anastomosis, as

$$
o_{p r}(\mathbf{x}, t)= \begin{cases}s_{o_{p r}} & \text { if } \quad \tau\left(\Sigma_{\sigma(\mathbf{x})}\right)=Q ; \\ s_{o_{p r}}^{\text {loop }} & \text { if } \quad \tau\left(\Sigma_{\sigma(\mathbf{x})}\right)=\{T, S\} \\ & \text { and } \Sigma_{\sigma(\mathbf{x})} \text { is within a loop; } \\ 0 & \text { otherwise. }\end{cases}
$$

The assumption that only cell sprouts forming loops are able to supply oxygen is realistic, because they have the functional capacity of maturing and carrying a flow of blood. In particular, closed loops are identified by an imaging algorithm that, at each MCS, tracks contiguous paths connecting the center of mass of adjacent vascular cells. Finally, we set $s_{o_{p r}}>s_{o_{p r}}^{\text {loop }}$ as biological evidence has widely provided that oxygen partial pressure in functional preexisting venules is higher than oxygen partial pressure in emerging capillaries [47].

We can finally give some analytical results on the system of RD equations (12)-(14) equipped, as we will see in the following section by no flux (Neumann) boundary conditions:

Proposition 2.1. (i) The fields $v, c, o_{p r}: \Omega \times[0, T] \rightarrow \mathbb{R}$, where $T$ is a final given time (possibly $+\infty$ ), are continuous in time and piecewise continuously differentiable in space (i.e., $\left.v, c, o_{p r} \in P C^{1}(\Omega,[0, T])\right)$;

(ii) Equations (12)-(14) preserve positivity, i.e., $v(\boldsymbol{x}, 0), c(\boldsymbol{x}, 0), o_{p r}(\boldsymbol{x}, 0) \geq 0 \forall \boldsymbol{x} \in \Omega \Longrightarrow$ $v(\boldsymbol{x}, t), c(\boldsymbol{x}, t), o_{p r}(\boldsymbol{x}, t) \geq 0 \quad \forall(\boldsymbol{x}, t) \in \Omega \times[0, T]$.

Proof. (i) It follows from the fact that the decay terms are continuous in time and space, while the uptake/consumption terms and the production terms are continuous in time and piecewise continuous in space (accounting, in particular, the possibility that secretion and/or uptake/consumption occur in specific regions of the domain and vanish elsewhere, as determined by the Kronecker deltas).

(ii) From the theory of parabolic equations, an RD equation preserves the positivity if the production terms are positive semidefined functions and the decay terms go to zero as the corresponding variable goes to zero, which 
holds in the case of our interest. Further, the uptake functions are here linear for small values of the concentrations allowing the application of classical results.

\section{Results}

Simulation details and parameter estimates. The simulation domain $\Omega$ is a square lattice of $1000 \times 1000$ sites. The characteristic size of each site is $1 \mu \mathrm{m}$, and therefore $\Omega$ represents a tissue with an area of $1 \mathrm{~mm}^{2}$. One MCS is set to correspond to $20 \mathrm{sec}$. This temporal correspondence is obtained by an empirical comparison between the outcomes of a preliminary set of simulations, focused on the chemotactic movement of a single tip cell, and specific data on the EC autonomous motility performed on embryonic mouse allantoides [46] (such a calibration is analogous to those presented in $[53,59]$ ). As a result, our virtual tip individuals are observed to move at an average speed of $\approx 20 \mu \mathrm{m} / \mathrm{h}$, which is also consistent with the values measured in [50]. The overall CPM simulations then are stopped after 10000 MCS, so that they reproduce a time-lapse of nearly 72 hours (3 days).

The PDEs describing the evolution of the molecular variables are numerically solved with a finite difference scheme on grids with the same spatial resolution as $\Omega$, characterized by 30 diffusion steps per MCS. This temporal scale is sufficiently small to guarantee the stability of the numerical method. Procedurally, after the discrete CPM has evolved through a MCS, the continuous equations are rederived, based on the new distribution of the cells, and solved. We use no flux boundary conditions.

All the model parameters are listed in Tables 1 and 2. In particular, the coefficient regulating molecular dynamics, as long as the threshold of phenotypic transitions, have been inferred by the experimental literature. The parameters describing the biophysical properties of the cells, such as their basal motility, elasticity and adhesive strengths (whose one-to-one correspondence with measurable quantities is not straightforward) have been instead evaluated, consistently with the biological considerations clarified along the text, through preliminary simulations or taking advantage of selected sensitivity analysis performed in CPM works dealing with vascular progression.

Simulation results. As represented in Fig. 1, we start with a preexisting vasculature, whose topology however does not allow a sufficient oxygenation of the overall tissue. The preexisting vessels are in fact not completely func- 
tional, as they are located too far with respect to the characteristic oxygen diffusion distance [11]. The tissue is initially seeded with a homogeneous oxygen partial pressure, that is sufficiently high to avoid an immediate transition to the hypoxic state (i.e., $o_{p r}(\mathbf{x}, 0)=o_{p r, i}>o_{p r, h}$ for all $\mathbf{x} \in \Omega$ ). On the contrary, we assume null amounts both of tissue-secreted VEGF-A and of EC autocrine chemical (i.e., $v(\mathbf{x}, 0)=c(\mathbf{x}, 0)=0$ for all $\mathbf{x} \in \Omega$ ). Indeed, all the vascular cells have initially a quiescent phenotype.

The tissue takes almost 6 hours to consume the initial reserve of oxygen, that can be no longer supplied by the vasculature (see Fig. 2). The subsequent production of VEGF-A is indeed the tissue response to hypoxia. VEGF-A activity then starts promoting the activation of most endothelial cells located at the vessel walls, as captured by the corresponding graph in Fig. 3. Such individuals partially lose their adhesive interactions and differentiate, according to the notch-mediated selection and lateral inhibition, into either the tip or the stalk fate. This process generates an alternating, i.e., salt-and-pepper, pattern of cell phenotypes, where tip cells are separated by small clusters of stalk individuals (see Fig. 4 (a)). Tip cells then chemotactically migrate along gradients of tissue-secreted VEGF-A, while vascular sprouts grow behind them (due to the remaining but sufficient intercellular adhesion). Sprout elongation and reinforcement are mainly due to stalk cell proliferation. Tip ECs can differentiate also within growing sprouts, leading to further branching phenomena, as represented in Fig. 4 (c). The overall formation of a capillary-like network is guided by the morphogenic activity of the EC autocrine growth factor. In particular, at the end of the simulation, a structured vascular plexus, where cords of cells enclose lacunae, covers the entire tissue, see the last panel of Fig. 1. More in detail, capillary branches typically are one or two cells wide, while lacunae, characterized by $5-6$ constituent nodes, reach a roughly uniform size over time, after that the smaller ones shrink and disappear (cf. Fig. 3). Such characteristic measures agree well with the corresponding values reported in the experimental literature $[17,42,60]$.

The emerging network is instrumental for an optimal metabolic exchange: it can in fact provide a sufficient and almost uniform oxygen supply to the entire tissue, that indeed recovers an oxygenated state and no longer produces VEGF-A, see Figs. 2 and 3. This results in the late inactivation of most endothelial cells (i.e., which return to the quiescent state, as captured by the corresponding graph in Fig. 3), that are stabilized in a functional structure that, without the necessity of significant further movement, is in principle 
able to differentiate to form mature vessels.

Analysis of the model components and of potential causes preventing a correct tissue reoxygenation. In the previous part of the section, we have demonstrated the model consistency in reproducing the vascularization of a hypoxic tissue. Indeed, we are now in the position to dissect the component mechanisms of the angiogenic process, in order to analyze the emergence of abnormal capillary networks due to altered cell behavior. This study is of particularly importance from a biomedical viewpoint, as, in principle, it allows to identify potential causes (and therefore selected targets for intervention strategies) which prevent to assure a complete reoxygenation of hypoxic tissues.

The directionally preferential stalk cell mitosis is fundamental for an optimal sprout extension. As represented in Fig. 5 (top panels), the detailed process is the following: i) a stalk cell divides perpendicular to the direction of sprout growth; ii) its two daughter cells, maturating into full-size individuals, than push the leading part of the sprout, which therefore extends into the extracellular space; iii) the elongation of the sprout is eventually sustained by the proliferation of other component stalk cells. A fail in the intracellular regulatory mechanisms, which control the correct spatial disposition of the daughter cells, can cause serious malformations in the growing vascular capillaries. For instance, if stalk individuals are allowed to divide along a random axis, the probability of the emerging of short and swollen sprouts, which extends in width and not in length, dramatically increases, see Fig. 5 (middle panel). With a biological viewpoint, we can observe that, in physiological conditions, the direction of cell duplication is defined by the orientation of the mitotic spindle, the microtubule structure that attaches chromosomes allowing their correct segregation. The axes of the mitotic spindle and cell division are in fact perpendicular to each other. All mechanisms behind the formation and the orientation of the mitotic spindle are not clear yet: however, it worth hypothesize that the positioning of the mitotic spindle is determined by specific stresses acting on cell membranes and transmitted by the actin cytoskeleton [35]. In the case of the proliferation of stalk cells, such mechanical signals are determined by the extension of the sprout they belong to [20]. In this respect, we can therefore speculate that serious malformations in growing capillaries may derive from an incorrect positioning of the spindle of the component stalk individuals, which may be not correlated with the directionality of the stretches acting on their membrane. 
The overall inhibition of stalk cell proliferation produces instead stunted vascular sprouts, that are not able to further elongate, i.e., remaining formed by a tip cell and by 1-2 stalk individuals. Such a poor branches quickly join each other giving rise to loop structures characterized by an intercapillary distance too low for a functional transport and diffusion of oxygen, see the bottom panels of Fig. 5. Abnormal mitotic processes for stalk cells may be related to disruption of some mitotic check points. In fact, as widely known from the biological literature, each step of mitosis is highly regulated. In particular, a very critical stage, that typically delays or inhibits at all cell proliferation, is the correct alignment of chromosomes, which is mandatory for the cell to equally divide its chromosomes between the two daughters to guarantee the correct transmission of genetic material [30]. This step is controlled by the spindle assembly checkpoint (SAC), a highly organized mechanism formed by several different proteins that does not allow cell division to proceed unless each pair of chromatids is attached to opposite spindle poles.

A complete vascularization of the tissue can be prevented by the inhibition of the cell chemotactic response, implemented in the model by setting $\mu_{c}=$ $\mu_{v}=0$ in Eq. (10). Such an alteration in cell physiology implies that both tip and stalk individuals lose their directional guidance cues. The result is that growing sprouts elongate in random directions, which are uncorrelated from the chemical gradients (and therefore from the hypoxic areas of the tissue). In particular, as the leader tip individuals maintain a degree of directional persistence, due to term (3), some capillary branches may even organize in self-loops or rejoins with the preexisting vessel (see Fig. 6 (top panels)). These structures are obviously unable to transport oxygen (and nutrients): they represent only vascular appendage deprived of any physiological role. Biologically, the inhibition of the cell chemotactic migration can be caused either by a pathological downregulation and/or a dysfunction of the cell membrane receptors (for example, VEGF tyrosine kinase receptors) or by the presence in the extracellular space of saturating amounts of angiogenic factors, which therefore extinguish morphogen gradients (as experimentally provided in [60]).

The formation of a functional vascular bed can be disrupted also by an interference in the delta-notch signaling pathways that, as seen, control the correct tip cell selection and lateral inhibition. By excluding this mechanism from the model, we observe the emerging of a huge number of stunted sprouts, formed by only 1-2 elongated tip cells. Being too close each other 
(since there are not stalk individuals in between to assure a sufficient interbranch distance), such minimal vascular extensions quickly collapse, forming thick clumps of tip cells (see Fig. 6 (bottom panels)). Interestingly, partial and full inhibition of delta-notch regulatory cascade can be used as a therapeutic strategy both for retinal vasculopathies [26] and to prevent tumor vascularization $[43,49]$.

We finally study the effects on the angiogenic process of the size of the hypoxic region of the tissue. To this purpose, we maintain the same domain $\Omega$ (as well as the same model components and parameters), but we assume that the preexisting vasculature is only peripheral, i.e., located along the domain boundary. The hypoxic area of the host tissue is therefore much more extended with respect to the previous sets of simulations. Nevertheless, the tissue is again completely vascularized and reoxygenated, although the overall process requires a significant longer time, more than 5 days $(\approx 120$ hours), see Fig. 7 (top panels). Interestingly, the resulting capillary-like network is characterized by comparable topological properties (mean cord length, lacunae size and nodes per lacuna) of the vascular pattern previously obtained with a reduced tissue hypoxic area, as it is possible to observe by comparing the graphs in Fig. 3 and in Fig. 7. We can indeed conclude that the extension of the host tissue does not affect the angiogenic process in terms of vessel morphology and/or organization, but it only determines the time needed for the emergence of a structured and functional vascular bed, i.e., able to supply the oxygen to the entire hypoxic area.

\section{Discussion}

Angiogenic progression is an intrinsically multiscale program that consists of the formation of new capillaries from a preexisting functional vasculature. It is a process characteristic of many physio-pathological situations, such as the vascularization of solid tumors, of hypoxic tissues, of ovary and uterus during the female cycle, of mammary gland during lactation, and of granulation tissue during wound healing. It is also involved in some chronic inflammatory diseases like rheumatoidarthritis and psoriasis, in vasculopathies like diabeticmicroangiopathy, in degenerative disorders like atherosclerosis and cirrhosis, and in tissue injury occurring in ischemia. Indeed, a deep under-

standing of the underlying component mechanisms represents a tremendous challenge for both experimental and computational researchers. 
In this respect, in this paper we have presented a multilevel CPM-based approach describing selected dynamics of an in vivo vascularization, and subsequent reoxygenation, of a hypoxic tissue. The proposed model has been able to link the mesoscopic cell phenomenology to selected microscopic intracellular pathways. Moreover, as a distinct feature of our approach, we have focused on the role played in the angiogenic process by the behavioral differentiation of both vascular and of tissue cells. In particular, the preexisting mature capillaries are assumed to be formed of quiescent ECs, that are then activated by tissue-secreted VEGF and differentiate in tip and stalk individuals according to a simplified delta-notch signaling cascade. Capillary-like pattern formation is then driven by the morphogenic activity of an ECreleased short-diffusing angiogenic factor, by the persistent migration of tip individuals and by the directionally-preferential division of stalk cells. The resulting model has shown a good degree of realism in reproducing the formation via sprouting angiogenesis of a functional network, characterized by specific geometrical properties, able to provide oxygen transport to the host. It is also useful to underline that our approach has been able to capture branching and anastomosis of growing vascular sprouts without any additional ad hoc rules. Finally, simulation results have predicted that abnormal capillary-like bed formations are caused by the abrogation of cell chemotactic response, as well as altered tip cell selection and stalk cell proliferation. Such model outcomes could in principle give rise to potentially efficient biomedical strategies that aim at reestablishing a correct tissue vascularization.

Model refinement and improvements. The proposed approach is characterized by many relevant assumptions and limitations, that can be improved in several directions. First of all, we have assumed that tip cells maintain their fate for the duration of the entire vascular elongation or until anastomosis events. However, it is useful to underline that few recent in vivo and in vitro studies have shown that, at least in some situations, a dynamic and continuous phenotypic interchange between stalk and tip individuals may also occur $[3,5,28]$. The hypotheses underlying the role played by the two VEGF isoforms are of course a simplified setting of the "real" physiological picture, although they are commonly accepted and used in the computational literature. In selected conditions, both short- and long-range diffusing VEGFs may in fact act in a paracrine fashion during sprouting angiogenesis. In particular, short-diffusing VEGF isoforms (such as VEGF-A $\mathrm{V}_{165}$ ) may be also expressed by non-vascular cells under hypoxia. 
Further, we have allowed oxygen transport thought emerging vascular loops without modeling the process of maturation and lumen formation. Simplifications have been made at the molecular level to limit the number of biochemical interactions as well. We have in fact neglected specific subcellular regulatory pathways that underlie both the hypoxic transition of tissue cells (HIF-dependent cascades) and the VEGF-induced activation of endothelial individuals. In particular, angiogenic factors have been widely demonstrated to activate a number of downstream intracellular cascades which control the behavior and the biophysical properties of vascular cells [33]. Among them, a fundamental role is played by calcium signaling pathways, as reported in great details in the experimental literature, see for instance $[40,41,42]$ and references therein.

A further, natural development of the proposed approach could be the incorporation of a description of the kinetics of the cytoskeletal remodeling. The present version of the model in fact does not focus on the dynamics of actin filaments, but rather considers the cell body as a single and homogeneous elastic element undergoing local mechanical stresses on its membrane, due to thermodynamic forces and chemical stimuli. A more detailed multiscale model would use the stress distributions at the plasma membrane (PM) as a signaling input for the subsequent polymerization process of intracellular cytoskeletal units. Moreover, the introduction of cytoskeletal dynamics would also result in a more accurate description of the mechanisms underlying the directionally-preferential division of stalk individuals.

However, according to us, the fundamental improvement of our model is the explicit introduction of the interactions between endothelial cells and extracellular environment, in particular with the extracellular matrix (ECM) characterizing the host tissue. The ECM, which forms the architecture of a number of structures, i.e., two-dimensional (2D) flat basement membranes, or three-dimensional (3D) connective tissues, can be described as a complex network of insoluble structural fibrous proteins such as different types of collagen, fibrins and fibronectins, mixed with soluble glycosaminoglycans and glycoproteins. The ECM is therefore highly heterogeneous in both density, microstructural properties (i.e., stiffness) and orientations, with irregular concentrations of the component proteins. Furthermore, matrix determinants strongly differ in healthy and damaged tissues as well; in particular, specific ECM microenvironments characterized distinct types of tumor. The ECM provides mechanical support to cells and mediates intercellular signaling, which can act over sufficiently long time and distances, via secreted 
molecules and mechanical stresses and strains. The ECM can also affect cell motility according to several mechanisms. First, crawling cells can follow local gradients of ECM protein concentrations, a process called haptotaxis. Such gradients can be either preexisting within the matrix or generated via deposition or degradation of matrix components. In this respect, it is widely known that angiogenic factors stimulate tip cells to produce proteolytic enzymes (for example, matrix metalloproteinases, MMPs), which digest matrix proteins around the leading edge of the sprout thereby creating guidance cues for further branch elongation [34, 45]. Cell migratory ability can be also determined by the absolute concentrations of ECM components: this mechanism is called haptokinesis. At this regard, several experimental studies show that cell speed, spreading, and membrane activity is maximal at intermediate levels of ECM densities, both on 2D substrates [14, 19] and in 3D matrices [73]. Also the local rigidity of the matrix plays a fundamental role in determining the efficiency and the direction of cell crawling, e.g. the so-called durotaxis, see again [73] and references therein. We also mention that cell-matrix adhesion is a further determinant in cell migration: as provided by innumerable studies [7, 18, 44, 73], an overly adhesive ECM causes in fact the formation of clusters of integrin-ligand bounds at the PM of the cells, that are therefore not able to detach and further migrate. On the contrary, a too weak cell-ECM adhesion results in the packing of cells in big islands, as they try to minimize the part of their membrane in contact with the extracellular environment. However, for the sake of completeness, we recall that some authors have speculated that the contact interactions between vascular cells and the surrounding matrix environments play a major role in the stabilization of a vascular network (preserving its morphology under shear stresses and perturbations), rather than in its early formation, at least in in vitro systems [2].

Comparison with the pertinent literature. Because of the spatial scales involved, cell-based models are particularly suited to reproduce the formation of capillary patterns. In this respect, CPM-based approaches have investigated the main aspects and processes characterizing vascular systems. First of all, different published CPMs have focused on in vitro vasculogenesis, providing that relatively simple cell-level mechanisms, such as contact-inhibited chemotaxis [37], polarization in response to growth factor stimulations [38] and preferential adhesion to elongated structures [66, 67], are sufficient to obtain the organization of endothelial cell aggregates or dispersed popula- 
tions into bidimensional networks. In another interesting CPM-based model [61], the authors analyze how variations in cell-cell and cell-matrix adhesive interactions result in different patterns of a cluster of malignant cells, seeded within a 3D matrix and stimulated by a chemical substance. In particular, they find that spreading of individual cells is obtained with a low intercellular adhesion. On the opposite, a high cell-cell contact strength is able to maintain the compactness of the cell colony. Finally, if both cell-cell and cell-matrix adhesive interactions fall in the same intermediate range of values, tubular structures emerge (only with the inclusion of a contact-inhibition of chemotaxis). The authors then use their model results to predict the efficacy of some therapeutic interventions, able to interfere with the evolution of the malignant cell colony. In [32], a more complex CPM reproduces an in vivo vasculogenic process during embryonic development, where endothelial precursor cells of mesodermal origin, known as angioblasts, assemble into a characteristic network pattern. In particular, the authors assume that VEGF is not autocrinally produced by the angioblasts, but by the ECM. The EC precursors instead secrete non-diffusive matrix components, able to bind and immobilize the signalling agent in the cell close proximity. However, the works cited so far have approached the biological problem only from a phenomenological view point, prescribing to the ECs a set of rules to follow during the entire patterning. It has been therefore neglected the evolution of the internal state of the cells and of the intracellular dynamics that underlie their phenotypic behavior. In [56, 57], an extension of CPMs in this direction takes into account specific VEGF-induced calcium-dependent pathways, that regulate the early stages of the vasculogenic process of a dispersed population of tumor-derived endothelial cells (TECs). In particular, the biochemical state of each cell is assumed to establish its specific biophysical properties, that therefore are no longer constant over time or homogenous within the entire cell body, but constantly adapt during the process, as a consequence of continuous internal and external stimuli.

CPM-based approaches have also dealt with vascular angiogenesis. In [12], the authors present a CPM pointing out that branched vascular trees emerge from a preexisting vessel and chemotactitally grow toward an external source of VEGF guided by haptokinetic and haptotactic mechanisms (ECM gradients are created by cell proteolysis) and sustained by cell proliferation. If in this approach and in [32] the ECM is described as a homogenous field, the model in [4] analyzes the tumor-induced angiogenesis representing also the fibrous component of the matrix, that is assumed to act as a rigid ob- 
stacle influencing cell migration. However, all the CPM commented so far neglect the differentiation between vascular cell phenotypes (i.e., tip, stalk, quiescent). Such a model component is present in [62], as the authors identify three types of tumor cells, i.e., normal, hypoxic and necrotic and three of endothelial cells i.e., vascular, inactive neovascular and active neovascular. In particular, vascular cells build the preexisting mature capillaries, while neovascular cells, which behave exactly as the vascular ones, can irreversibly switch into active neovascular cells after agonist stimulation. Active neovascular cells can proliferate and chemotax up gradients of VEGF-A. In more details, active neovascular individuals are characterized by a behavior that is a mix of the phenotypes of both tip and stalk cells: in fact, they respond to tumor-secreted morphogen gradients, dragging the rest of the sprout, but they are also allowed to proliferate. In this respect the elongating branches are formed by only active neovascular individuals. It is also useful to notice that, with respect to our model, in [62] the VEGF activity is not responsible for the activation of quiescent cells forming the preexisting vessels, as inactive vascular individuals are a priori located within mature capillaries.

Conclusions. This paper has presented a cellular Potts model able to realistically reproduce an in vivo vascularization, and the subsequent reoxygenation, of a hypoxic tissue. As a key feature of our approach, vascular cells dynamically differentiate from a quiescent state to either a stalk or a tip phenotype, upon VEGF activity and according to the well-known deltanotch signalling pathway. Such phenotypic transitions, along with chemotactic mechanisms and a proper directionality of stalk cell mitotic events, have been here provided to be fundamental for the emergence of a functional capillary network, i.e., able to effectively supply oxygen to the tissue. In this respect, the proposed model represents a refinement of a series of published CPMs, that deal with different scenarios of vascular progression, i.e., in vitro tubulogenesis $[25,37,38]$, endothelial cell collective migration and patterning [66, 67, 68], tumor-induced angiogenesis [62]. However, our CPMbased framework can be further improved in several directions: for instance, it would be interesting to analyze the effect on vascular extension of selected mechanical and chemical stimuli that may derive both from the extracellular environment (i.e., insoluble matrix components) and from other diffusive substances (i.e., other growth factors or VEGF isoforms). 


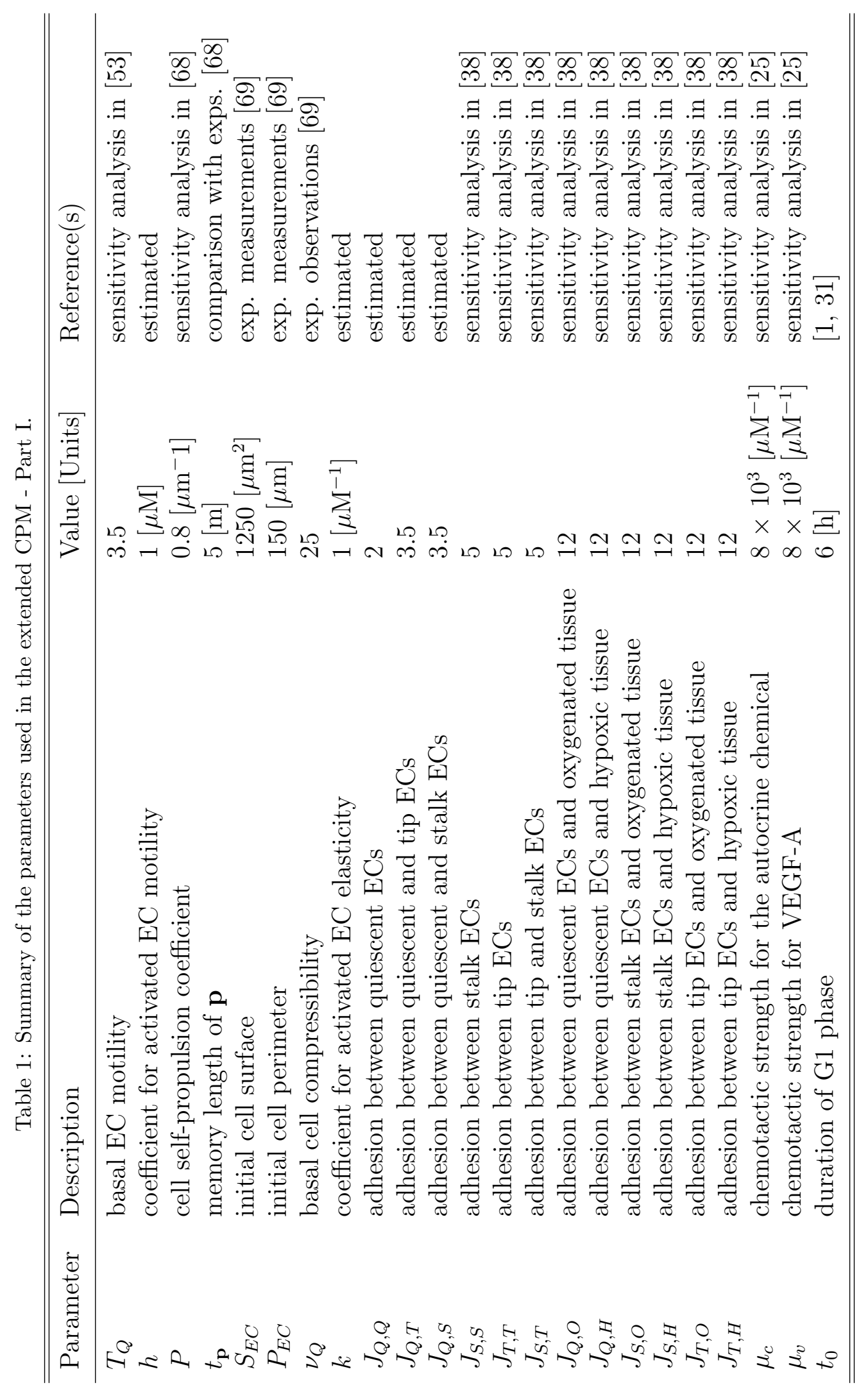




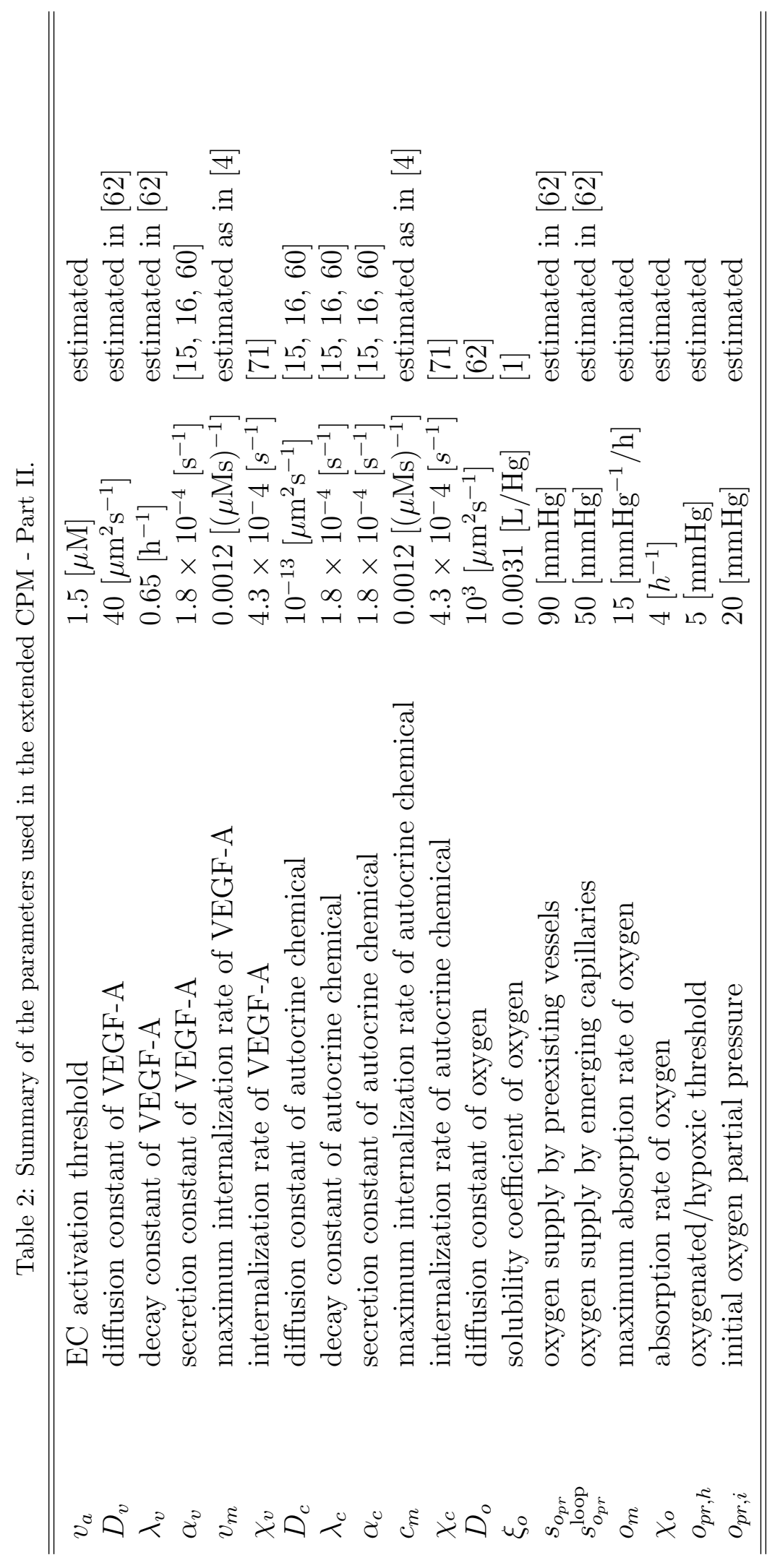


[1] Alberts B, Johnson A, Lewis J, Raff M, Roberts K, Walter P. Molecular Biology of the Cell, 3rd ed. Garland Science; 2002.

[2] Ambrosi D, Gamba A, Serini G. Cell directional persistence and chemotaxis in vascular morphogenesis. Bull Math Biol, 2004; 66: 1851 - 73.

[3] Arima S, Nishiyama K, Ko T, Arima Y, Hakozaki Y, Sugihara K, Koseki H, Uchijima Y, Kurihara Y, Kurihara H. Angiogenic morphogenesis driven by dynamic and heterogeneous collective endothelial cell movement. Development, 2011; 138: 4763 -76 .

[4] Bauer AL, Jackson TL, Jiang Y. A cell-based model exhibiting branching and anastomosis during tumor-induced angiogenesis. Biophys J, 2007; 92: $3105-21$.

[5] Bentley K, Franco CA, Philippides A, Blanco R, Dierkes M, Gebala V, Stanchi F, Jones M, Aspalter IM, Cagna G, Weström S, Claesson-Welsh L, Vestweber D, Gerhardt H. The role of differential VE-cadherin dynamics in cell rearrangement during angiogenesis. Nat Cell Biol, 2014; 16: $309-21$.

[6] Bussolino F, Arese M, Audero E, Giraudo E, Marchio S, Mitola S, Primo L, Serini G. Biological aspects in tumor angiogenesis. In Preziosi L, Ed. Cancer Modeling and Simulation, Mathematical Biology and Medicine Sciences, Chapman and Hall/CRC 2003; pp $1-16$.

[7] Burgess BT, Myles JL, Dickinson RB. Quantitative analysis of adhesion-mediated cell migration in three-dimensional gels of RGD-grafted collagen. Ann Biomed Eng, 2003; 28: $110-8$.

[8] Brooker R, Hozumi K, Lewis J. Notch ligands with contrasting functions: jagged1 and delta1 in the mouse inner ear. Development, 2006; 133: 1277 - 86.

[9] Carmeliet P, Jain RK. Angiogenesis in cancer and other diseases. Nature, 2000, 407, $249-57$.

[10] Carmeliet P. Angiogenesis in life, disease and medicine. Nature, 2005; 438: $932-6$.

[11] Chilibeck PD, Paterson DH, Cunningham DA, Taylor AW, Noble EG. Muscle capillarization $\mathrm{O} 2$ diffusion distance, and VO2 kinetics in old and young individuals. J Appl Physiol, 1997; 82: $63-9$.

[12] Daub JT, Merks RMH. A cell-based model of extracellular-matrix-guided endothelial cell migration during angiogenesis. Bull Math Biol, 2013; 75: 1377 - 99.

[13] De Smet F, Segura I, De Bock K, Hohensinner PJ, Carmeliet P. Mechanisms of vessel branching: filopodia on endothelial tip cells lead the way. Arterioscler Thromb Vasc Biol, 2009; 29: 639 - 49. 
[14] DiMilla PA, Stone JA, Quinn JA, Albelda SM, Lauffenburger DA. Maximal migration of human smooth-muscle cells on fibronectin and type-IV collagen occurs at an intermediate attachment strength. J Cell Biol, 1993, 122: 729 - 37.

[15] Distler JW, Hirth A, Stolarska MK, Gay RE, Gay S, Distler O. Angiogenic and angiostatic factors in the molecular control of angiogenesis. Q J Nucl Med, 2003; 47: $149-161$.

[16] Fernandez-Sauze S, Delfino C, Mabrouk K, Dussert C, Chinot O, Martin PM, Grisoli F, Ouafik L, Boudouresque F. Effects of adrenomedullin on endothelial cells in the multistep process of angiogenesis: involvement of CRLR/RAMP2 and CRLR/RAMP3 receptors. Int J Cancer, 2004; 108: 797 - 804.

[17] Fiorio Pla A, Grange C, Antoniotti S, Tomatis C, Merlino A, Bussolati B, Munaron L. Arachidonic acid-induced Ca2+ entry is involved in early steps of tumor angiogenesis. Mol Cancer Res, 2008; 6: 535 - 45.

[18] Friedl P, Entschladen F, Conrad C, Niggemann B, Zänker KS. CD4+ Tlymphocytes migrating in three-dimensional collagen lattices lack focal adhesions and utilize $\beta 1$ integrin-independent strategies for polarization, interaction with collagen fibers and locomotion. Eur J Immunol, 1998; 28: 2331 - 43.

[19] Gaudet C, Marganski WA, Kim S, Brown CT, Gunderia V, Dembo M, Wong JY. Influence of type I collagen surface density on fibroblast spreading, motility, and contractility. Biophys J, 2003; 85: 3329 - 35.

[20] Gerhardt H, Betsholtz C. How do endothelial cells orientate? EXS, 2005; 94: 3 15.

[21] Glazier JA, Balter A, Merks RMH, Poplawski NJ, Swat M. The Glazier-GranerHogeweg model: extension, future direction, and opportunitites for further study. In Anderson ARA, Chaplain MAJ, Rejniak KA, Eds. Single-Cell-Based Models in Biology and Medicine, Mathematics and Biosciences in Interactions. Birkaüser 2007; pp $79-106$.

[22] Glazier JA, Balter A, Poplawski NJ. Magnetization to morphogenesis: A brief history of the Glazier-Graner-Hogeweg model. In Anderson ARA, Chaplain MAJ, Rejniak KA, Eds. Single-Cell-Based Models in Biology and Medicine, Mathematics and Biosciences in Interactions. Birkaüser 2007; pp $79-106$.

[23] Glazier JA, Graner F. Simulation of the differential adhesion driven rearrangement of biological cells. Physical Rev E, 1993, 47: 2128 - 54.

[24] Graner F, Glazier JA. Simulation of biological cell sorting using a two dimensional extended Potts model. Phys Rev Lett, 1992; 69: 2013 - 17. 
[25] Guidolin D, Albertin G, Sorato E, Oselladore B, Mascarin A, Ribatti D. Mathematical modeling of the capillary-like pattern generated by adrenomedullin-treated human vascular endothelial cells in vitro. Dev Dyn, 2009; 238: 1951 - 63.

[26] Hellström M, Phng LK, Hofmann JJ, Wallgard E, Coultas L, Lindblom P, Alva J, Nilsson AK, Karlsson L, Gaiano N, Yoon K, Rossant J, Iruela-Arispe ML, Kaln M, Gerhardt H, Betsholtz C. Dll4 signalling through Notch1 regulates formation of tip cells during angiogenesis. Nature, 2007; 445: 722 - 3 .

[27] Ising E. Beitrag zur theorie des ferromagnetismus. Z Physik, 1925; 31: 253.

[28] Jakobsson L, Franco CA, Bentley K, Collins RT, Ponsioen B, Aspalter IM, Rosewell I, Busse M, Thurston G, Medvinsky A, Schulte-Merker S, Gerhardt H. Endothelial cells dynamically compete for the tip cell position during angiogenic sprouting. Nat Cell Biol, 2010; 12: 943 - 53.

[29] Jewell UR, Kvietikova I, Scheid A, Bauer C, Wenger RH, Gassmann M. Induction of HIF- $1 \alpha$ in 415 response to hypoxia is instantaneous. FASEB J, 2001; 15: 1312 4.

[30] Jia L, Kim S, Yu H. Tracking spindle checkpoint signals from kinetochores to APC/C. Trends Biochem Sci, 2013; 38: 302 - 11.

[31] Karn J, Watson JV, Lowe AD, Green SM, Vedeckis W. Regulation of cell cycle duration by c-myc levels. Oncogene, 1989; 4: $773-87$.

[32] Köhn-Luque A, de Back W, Starruss J, Mattiotti A, Deutsch A, Prez-Pomares JM, Herrero MA. Early embryonic vascular patterning by matrix-mediated paracrine signalling: a mathematical model study. PLoS One, 2011; 6: e24175.

[33] Kowanetz M, Ferrara N. Vascular endothelial growth factor signaling pathways: therapeutic perspective. Clin Cancer Res, 2006; 12: 5018 - 22.

[34] Lamalice L, Le Boeuf F, Huot J. Endothelial cell migration during angiogenesis. Circ Res, 2007; 100: 782 - 94.

[35] Lancaster OM, Baum B. Shaping up to divide: Coordinating actin and microtubule cytoskeletal remodelling during mitosis. Semin Cell Dev Biol, 2014; 34: 109 - 15.

[36] Liu ZJ, Shirakawa T, Li Y, Soma A, Oka M, Dotto GP, Fairman RM, Velazquez OC, Herlyn M Regulation of Notch1 and Dll4 by vascular endothelial growth factor in arterial endothelial cells: implications for modulating arteriogenesis and angiogenesis. Mol Cell Biol, 2003; 23: 14 - 25.

[37] Merks RMH, Perryn ED, Shirinifard A, Glazier JA. Contact-inhibited chemotactic motility: role in de novo and sprouting blood vessel growth. PLOS Comp Biol, 2008, 4: e1000163. 
[38] Merks RMH, Glazier JA, Brodsky SV, Goligorksy MS, Newman SA. Cell elongation is key to in silico replication of in vitro vasculogenesis and subsequent remodeling. Develop Biol, 2006; 289: $44-54$.

[39] Metropolis N, Rosenbluth AE, Rosenbluth MN, Teller AH, Teller E. Equation of state calculations by fast computing machines. J Chem Phys, 1953; 21: 1087 - 92.

[40] Munaron L. Intracellular calcium, endothelial cells and angiogenesis. Recent Patents Anticancer Drug Discov, 2002; 1: 105 - 19.

[41] Munaron L, Fiorio Pla A. Endothelial calcium machinery and angiogenesis: understanding physiology to interfere with pathology. Curr Med Chem, 2009; 16: 4691 703.

[42] Munaron L, Tomatis C, Fiorio Pla A. The secret marriage between calcium and tumor angiogenesis. Technol Cancer Res Treat, 2008; 7: 335 - 9.

[43] Noguera-Troise I, Daly C, Papadopoulos NJ, Coetzee S, Boland P, Gale NW, Lin HC, Yancopoulos GD, Thurston G. Blockade of Dll4 inhibits tumour growth by promoting nonproductive angiogenesis. Nature, 2006; 444: 1032 - 7.

[44] Palecek SP, Loftus JC, Ginsberg MH, Lauffenburger DA, Horwitz AF. Integrinligand binding properties govern cell migration speed through cell-substratum adhesiveness. Nature, 1997; 385: $537-40$.

[45] Pepper MS. Role of the matrix metalloproteinase and plasminogen activatorplasmin systems in angiogenesis. Arterioscler Thromb Vasc Biol, 2011; 21: 1104 -17 .

[46] Perryn ED, Czirok A, Little CD. Vascular sprout formation entails tissue deformations and VE-cadherin dependent cell-autonomous motility. Dev Biol, 2008; 313: $545-55$.

[47] Popel AS. Theory of oxygen transport to tissue. Crit Rev Biomed Eng, 1989; 17: $257-321$.

[48] Potts RB. Some generalized order-disorder transformations. Proc Camb Phil Soc, 1952; 48: $106-9$.

[49] Ridgway J, Zhang G, Wu Y, Stawicki S, Liang WC, Chanthery Y, Kowalski J, Watts RJ, Callahan C, Kasman I, Singh M, Chien M, Tan C, Hongo JA, de Sauvage F, Plowman G, Yan M. Inhibition of Dll4 signalling inhibits tumour growth by deregulating angiogenesis. Nature, 2006; 444: 1083 - 7.

[50] Rupp PA, Czirok A, Little CD. $\alpha \mathrm{v} \beta 3$ integrin-dependent endothelial cell dynamics in vivo. Development, 2004; 131: 2887 - 97. 
[51] Sainson RCA, Harris AL. Hypoxia-regulated differentiation: lets step it up a Notch. Trends Mol Med, 2006; 12: 141 - 43.

[52] Savill NJ, Hogeweg P. Modelling morphogenesis: from single cells to crawling slugs. J Theor Biol, 1997; 184: $118-24$.

[53] Scianna M. A Multiscale Hybrid Model for Pro-angiogenic Calcium Signals in a Vascular Endothelial Cell. Bull Math Biol, 2012; 74: 1253 - 91.

[54] Scianna M, Preziosi L. A hybrid Cellular Potts Model describing different morphologies of tumor invasion fronts. Math Model Nat Phenom, 2012; 7: 78 - 104.

[55] Scianna M, Preziosi L. Multiscale Developments of the Cellular Potts Model. Multiscale Model Simul, 2012; 10: $342-82$.

[56] Scianna M, Munaron L, Preziosi L. A multiscale hybrid approach for vasculogenesis and related potential blocking therapies. Prog Biophys Mol Biol, 2012, 106: 450 62 .

[57] Scianna M, Munaron L. Multiscale model of tumor-derived capillary-like network formation. Netw Heterog Media, 2011; 6: 597 - 624 .

[58] Scianna M, Bell CG, Preziosi L. A review of mathematical models for the formation of vascular networks. J Theor Biol, 2013; 333: 174 - 209.

[59] Scianna M. An extended Cellular Potts model analyzing a wound healing assay. Computers in Biology and Medicine, 2015; 62: $33-54$.

[60] Serini G, Ambrosi D, Giraudo E, Gamba A, Preziosi L, Bussolino F. Modeling the early stages of vascular network assembly. EMBO J, 2003; 22: $1771-9$.

[61] Singh J, Hussain F, Decuzzi P. The role of differential adhesion in cell cluster evolution, from vasculogenesis to cancer metastasis. Comput Methods Biomech Biomed Engin, 2015; 18: $282-92$.

[62] Shirinifard A, Gens JS, Zaitlen BL, Poplawski NJ, Swat M, Glazier JA. 3D multicell simulation of tumor growth and angiogenesis. PLoS One, 2009; 4: e7190.

[63] Smith JA, Martin L. Do cells cycle? Proc Natl Acad Sci U.S.A., 1973; 70: 1263 67.

[64] Steinberg MS. Reconstruction of tissues by dissociated cells. Some morphogenetic tissue movements and the sorting out of embryonic cells may have a common explanation. Science, 1963; 141: $401-8$.

[65] Steinberg MS. Does differential adhesion govern self-assembly processes in histogenesis? Equilibrium configurations and the emergence of a hierarchy among populations of embryonic cells. J Exp Zool, 1970; 173: 395 - 433. 
[66] Szabo A, Perryn ED, Czirok A. Network formation of tissue cells via preferential attraction to elongated structures. Phys Rev Lett, 2007; 98: 038102.

[67] Szabo A, Czirok A. The role of cell-cell adhesion in the formation of multicellular sprouts. Math Model Nat Phenom, 2012; 5: $106-22$.

[68] Szabo A, Varga K, Garay T, Hegedus B, Czirok A. Invasion from a cell aggregate: the roles of active cell motion and mechanical equilibrium. Phys Biol, 2012; 9: 016010 .

[69] Tomatis C, Fiorio Pla A, Munaron L. Cytosolic calcium microdomains by arachidonic acid and nitric oxide in endothelial cells. Cell Calcium, 2007; 41: 261 - 9.

[70] Turner S, Sherratt JA. Intercellular adhesion and cancer invasion: A discrete simulation using the extended Potts model. J Theor Biol, 2002; 216: 85 - 100.

[71] Wang D, Lehman RE, Donner DB, Matli MR, Warren RS, Welton ML. Expression and endocytosis of VEGF and its receptors in human colonic vascular endothelial cells. Am J Physiol Gastrointest Liver Physiol, 2002; 282: 1088 - 96.

[72] Williams CK, Li J-L, Murga M, Harris AL, Tosato G. Upregulation of the Notch ligand delta-like 4 inhibits VEGF induced endothelial cell function. Blood, 2006; 107: $931-9$.

[73] Zaman MH, Trapani LM, Sieminski AL, Mackellar D, Gong H, Kamm RD, Wells A, Lauffenburger DA, Matsudaira P. Migration of tumor cells in 3D matrices is governed by matrix stiffness along with cell-matrix adhesion and proteolysis. Proc Natl Acad Sci USA, 2006; 103: 10889 - 94. 


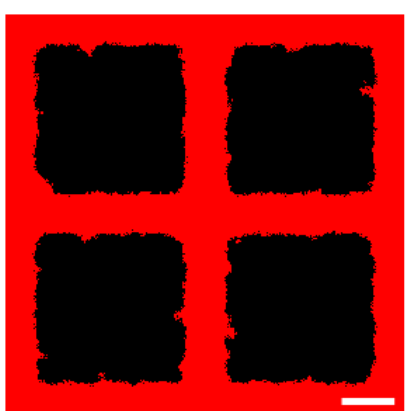

$1 \mathrm{~h}$

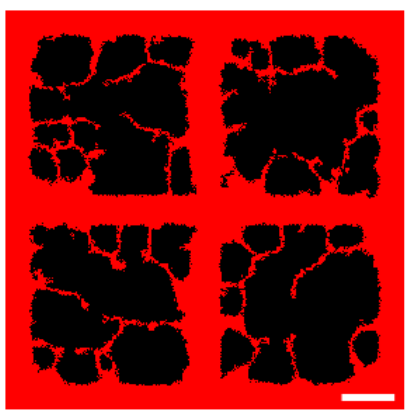

$45 \mathrm{~h}$

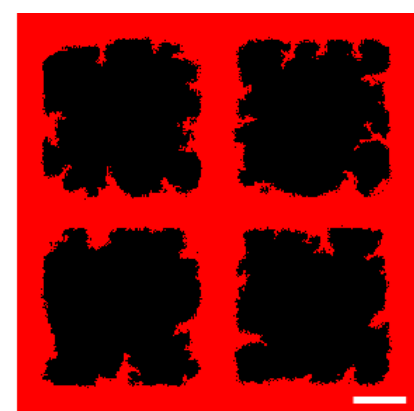

$15 \mathrm{~h}$

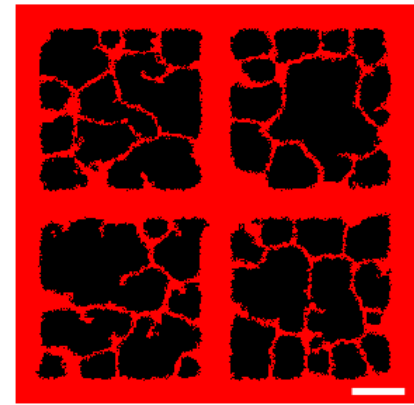

$60 \mathrm{~h}$

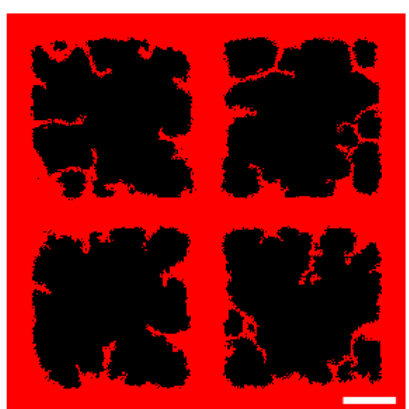

$30 \mathrm{~h}$

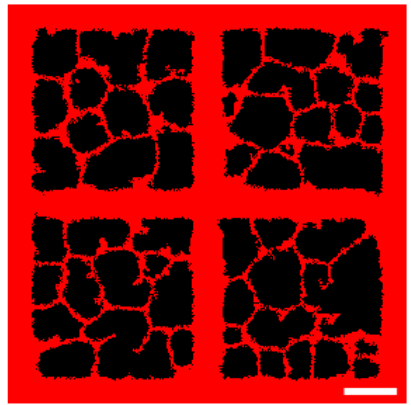

$72 \mathrm{~h}$

Figure 1: Model representation of the physiological angiogenic process. The initial condition consists of a preexisting vasculature too sparse to permit a complete oxygenation of the tissue, which quickly becomes hypoxic. Representative images show different stages of capillary-like network formation. All the model parameters are listed in Tables 12 . White scale bars: $100 \mu \mathrm{m}$. 


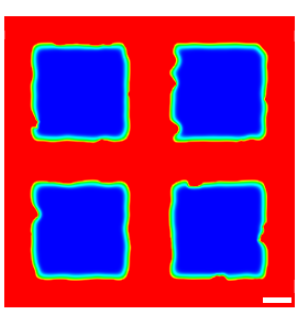

$6 \mathrm{~h}$

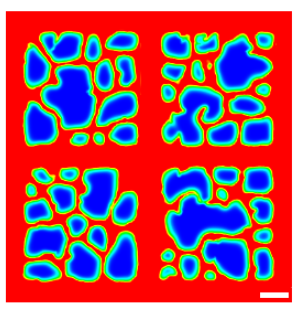

$60 \mathrm{~h}$

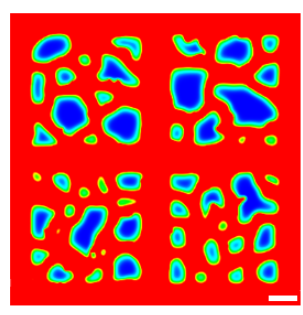

$66 \mathrm{~h}$

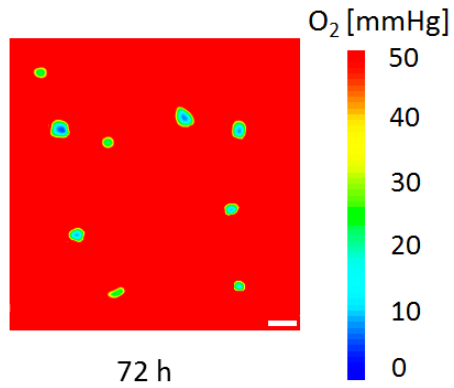

$72 \mathrm{~h}$

Figure 2: Representative images of the evolution of the oxygen partial pressure at different stages of the angiogenic process. $\mathrm{O}_{2}$ is supplied by quiescent vascular cells and by activated ECs that are within loops formed by anastomosis. White scale bar: $100 \mu \mathrm{m}$. 

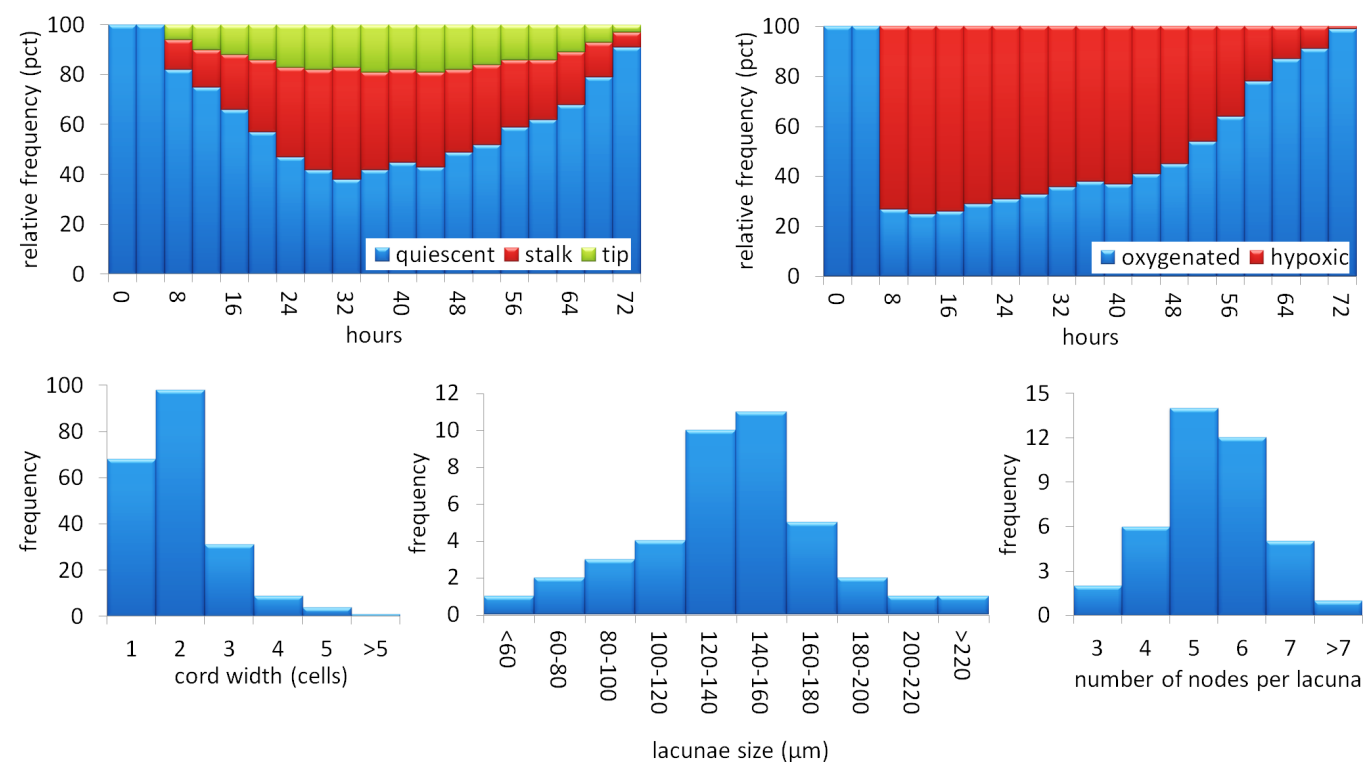

Figure 3: Top graphs: dynamical evolution of vascular and tissue cell phenotypes. It is possible to notice that, as the tissue becomes hypoxic, a consistent number of ECs differentiate, assuming either the stalk or the tip fate. However, in the final phases of the process, the tissue recovers an oxygenate state and therefore the vascular cells, no longer stimulated by VEGF-A, revert back to a quiescent condition. Bottom graphs: topological properties of the emerging vascular network. The final pattern is formed by lacunae with characteristic size $(\approx 120-160 \mu \mathrm{m})$ and number of nodes $(\approx 5-6)$, enclosed by 1-2 cell-wide cords. 


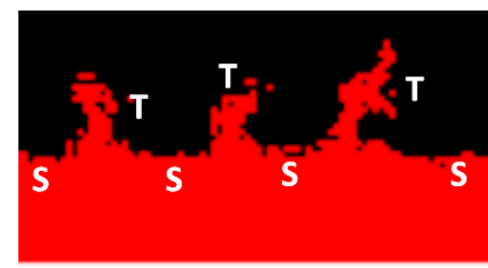

(a)

salt-and-pepper pattern
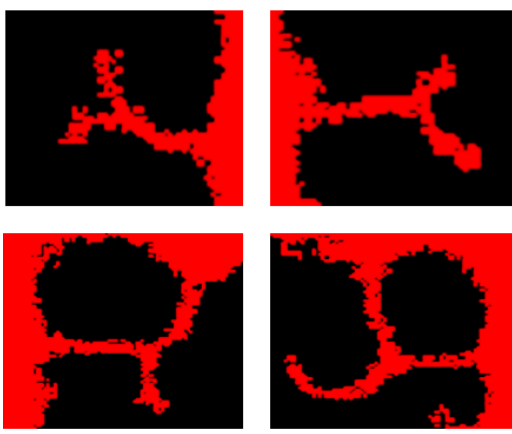

(b) branching morphogenesis

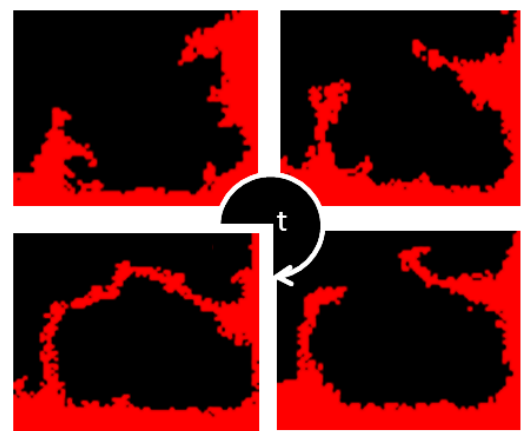

(c) anastomosis

Figure 4: Emerging phenomena during the angiogenic process. (a) Salt-and-pepper pattern resulting from the delta-notch mediated tip cell selection and lateral inhibition. (b) Branching morphogenesis can occur either during sprout growth (top panels) or after anastomosis (bottom panels). (c) Loop formation (anastomosis) is due to the collision and the subsequent adhesion of two separate sprouts (clockwise panels, as indicated by the white arrow). 

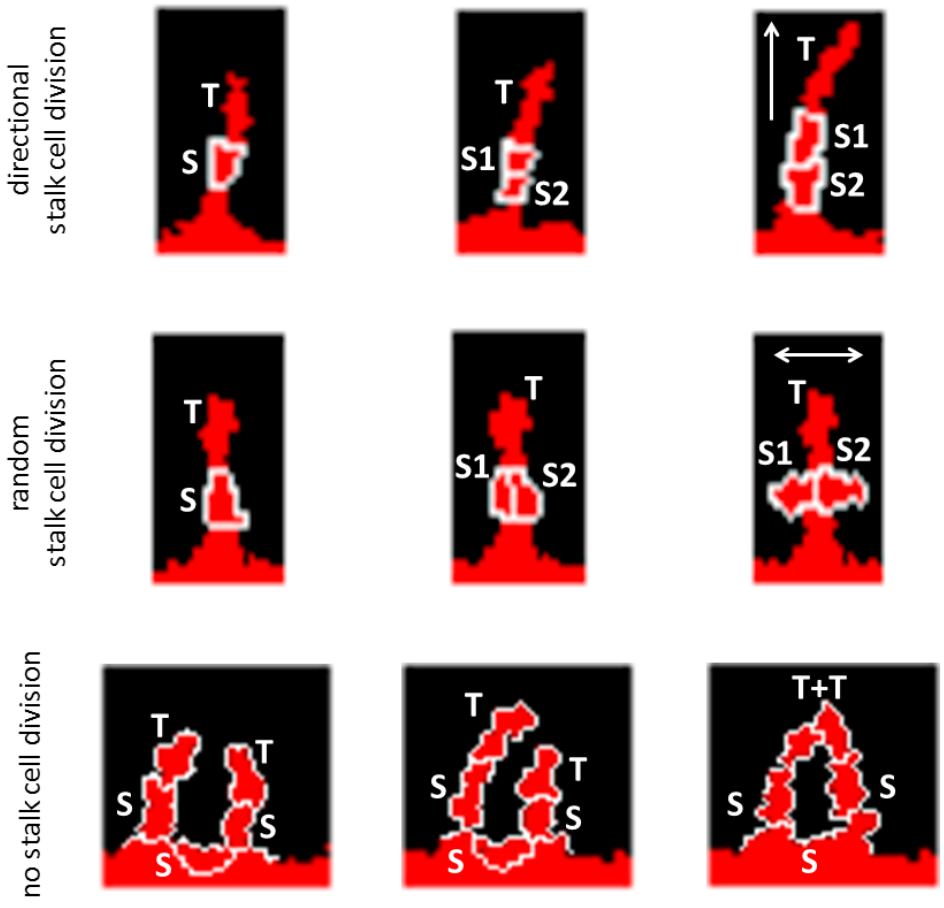

Figure 5: Role of stalk cell duplication in sprouting angiogenesis. Top panels: a directionally preferential mitosis allows a normal branch elongation. Middle panels: an altered duplication process, which does not identify a preferred axis for cell division, results in an abnormal sprout growth. Bottom panels: the inhibition of stalk cell proliferation finally causes the formation of short clumps of cells, which join in nonfunctional structures. For the readers' convenience cell membranes are depicted. 


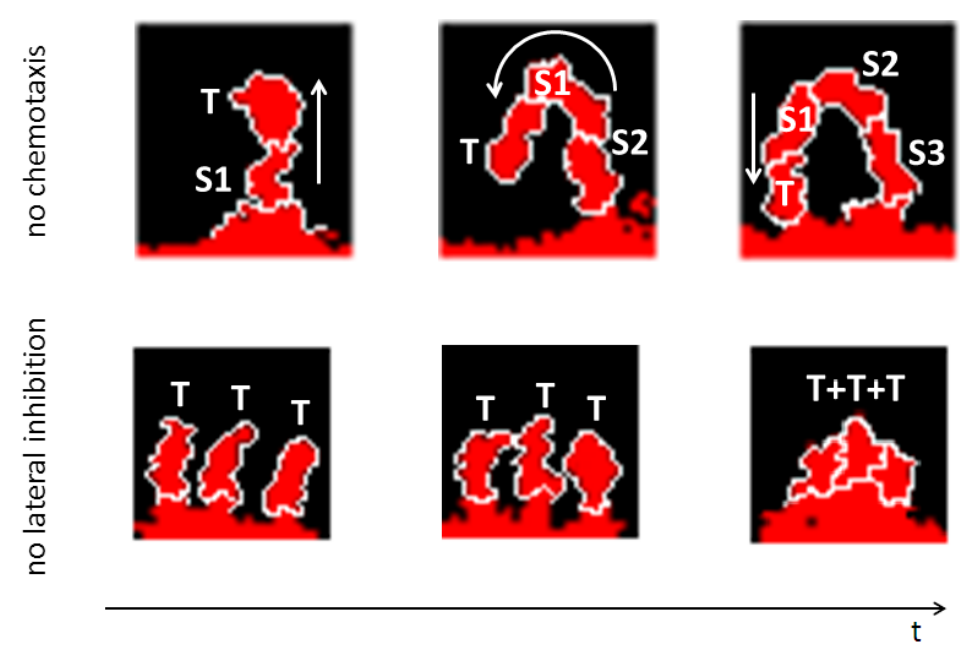

Figure 6: Role of other cellular mechanisms in sprouting angiogenesis. Top panels: the inhibition of the chemotaxis response for both tip and stalk cells results in the elongation of sprouts in random directions. Such branches do not grow along chemical gradients and therefore they do not extend towards hypoxic areas of tissue. Bottom panels: an interference in the delta-notch signalling pathways leads to the quick collapse of small groups of tip cells, which are distributed at insufficient distances along the preexisting vessel walls. For the readers' convenience cell membranes are depicted. 


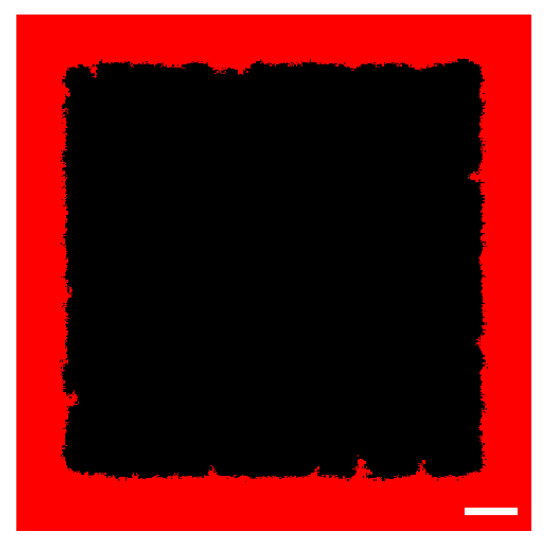

$1 \mathrm{~h}$

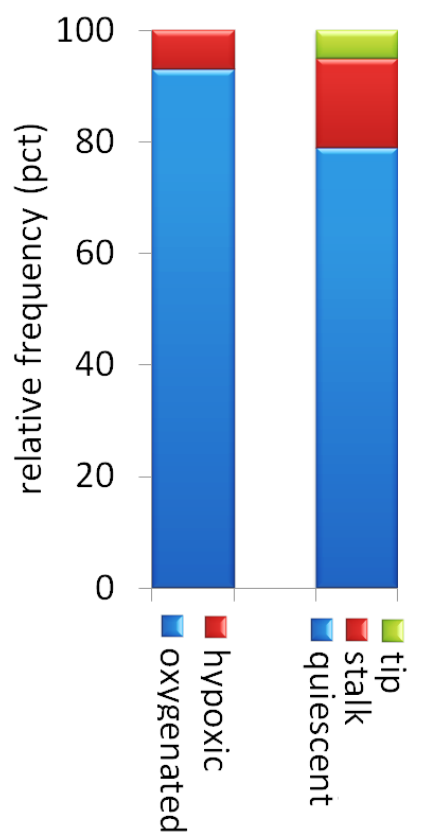

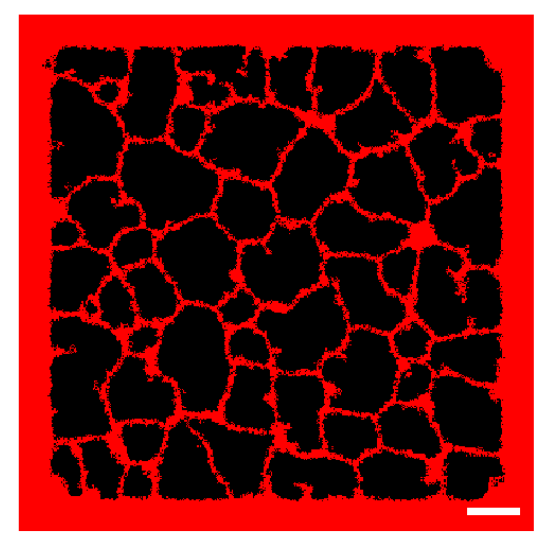

5 days
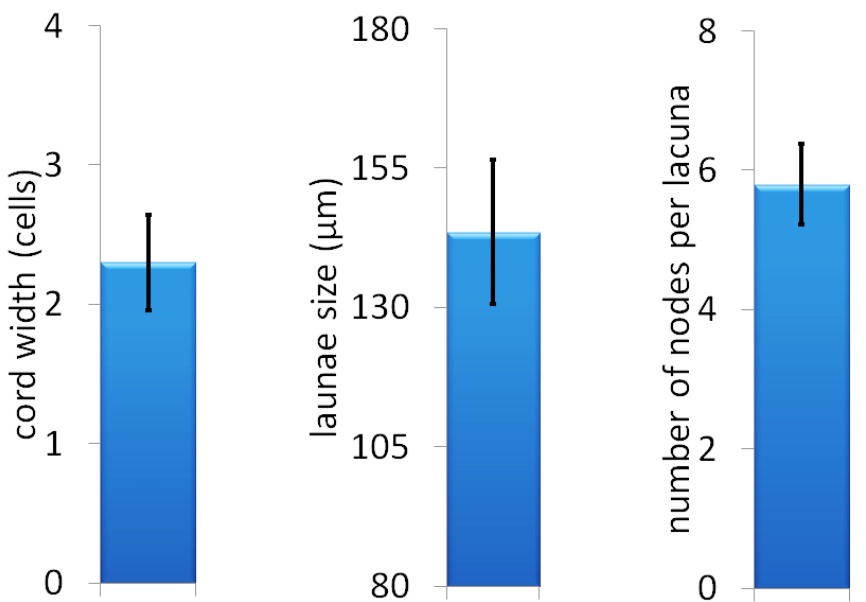

mean morphological dimensions

Figure 7: Model representation of the angiogenic process in the case of a peripheral preexisting vasculature (i.e., restricted along the domain border). The domain and the model parameters are the same as in the simulation setup proposed in Figs. 1 and 3. Top panels: tissue configuration at $1 \mathrm{~h}$ and $120 \mathrm{~h}(\approx 5$ days). White scale bars: $100 \mu \mathrm{m}$. Bottom plots: final (i.e., at $120 \mathrm{~h}$ ) relative percentage of each cell phenotype and characteristic measures of the resulting vascular network (values shown as mean $\pm \mathrm{sd}$ ). 